\title{
Strong-field ionization of laser-irradiated light homonuclear diatomic molecules: A generalized strong-field approximation-linear combination of atomic orbitals model
}

\author{
Vladimir I. Usachenko ${ }^{1,2, *}$ and Shih-I $\mathrm{Chu}^{2}$ \\ ${ }_{1}^{1}$ Institute of Applied Laser Physics UzAS, Nakashlyk Street, 1, Tashkent, 700185, Uzbekistan and Max-Born-Institute for Nonlinear Optics \\ and Short-Pulse Laser Spectroscopy, Max-Born-Strasse 2a, Berlin, 12489, Germany \\ ${ }^{2}$ Department of Chemistry, University of Kansas, Lawrence, Kansas 66045-7582, USA
}

(Received 16 September 2004; published 29 June 2005)

\begin{abstract}
The strong-field ionization in a number of light homonuclear diatomic molecules $\left(\mathrm{N}_{2}, \mathrm{O}_{2}\right.$, and $\left.\mathrm{H}_{2}\right)$ irradiated by an intense laser field of low fundamental frequency $\omega \ll I_{p}$ is considered theoretically and studied numerically compared to their "companion" atoms, having nearly identical ionization potential $I_{p}$. The background applied strong-field approach is based on using the $S$-matrix formalism of conventional strong-field approximation supplemented by the standard linear combination of atomic orbitals and molecular orbitals method utilized for approximate analytical reproduction of the two-centered wave function of an initial molecular bound state. Accordingly, the ionization of a diatomic molecule is described as a quantum-mechanical superposition (intramolecular interference) of contributions from ionization amplitudes corresponding to photoelectron emission from two atomic centers separated by equilibrium internuclear distance. Besides the bonding (or antibonding) symmetry of the highest occupied molecular orbitals (HOMO) corresponding to the outermost molecular valence shell, its spatial configuration and predominant orientation with respect to the internuclear axis and polarization of incident laser field also proved to be of substantial importance and, thus, are taken into equally detailed consideration. Moreover, wherever appropriate, the comparable contributions from other (inner) molecular valence shells of a larger binding energy (closest to that of HOMO, but of different bonding symmetry and spatial configuration) are additionally taken into account. The related results for calculated differential and/or integral molecular ionization rates, molecular photoelectron spectra, and angular distributions are fairly well consistent with available experimental data and, in particular, provide one with a transparent physical interpretation of the nature and origin of high suppression in ionization of the $\mathrm{O}_{2}$ molecule (as compared to its companion $\mathrm{Xe}$ atom) as well as no suppression in ionization of $\mathrm{N}_{2}$ molecules (as compared to its companion Ar atom).
\end{abstract}

DOI: 10.1103/PhysRevA.71.063410

PACS number(s): $33.80 . \mathrm{Rv}, 32.80 . \mathrm{Rm}, 34.50 . \mathrm{Gb}, 42.50 . \mathrm{Hz}$

\section{INTRODUCTION}

With the advent of powerful laser sources of intensities well over $\sim 10^{14} \mathrm{~W} / \mathrm{cm}^{2}[1,2]$ it has been possible to observe and study a variety of nonlinear fundamental strong-field phenomena [2-5] in laser-irradiated atomic and molecular species. Their nonlinear (multiphoton) character becomes manifest, in particular, by a highly nonlinear dependence of respective cross sections (or probabilities) and related observables on intensity $I$ of incident laser radiation. Thus, all these phenomena are substantially nonperturbative, insomuch as they cannot be adequately and entirely described yet by any finite-order perturbative expansion with respect to the electromagnetic (EM) interaction with incident laser field. Apart from pure fundamental interest, these processes offer good prospects for various practical applications, for example, the high-order harmonic generation (HHG) is currently believed to be highly promising for the creation of compact (tabletop) sources of ultrashort (subfemtosecond) pulses of powerful coherent xuv (x-ray ultraviolet) radiation [2,5]. Also, the intensity of very intense laser beams can be reliably calibrated by means of measuring the total yield of photoions produced due to multiphoton ionization (MPI),

\footnotetext{
*Corresponding author. Email address: vusach@yahoo.com
}

tunneling and/or above-threshold ionization (ATI). The latter two strong-field phenomena have been of great permanent research interest over the last two decades (e.g., [3-6] and also [7] for recent progress) because they particularly imply an absorption of many more photons than the minimum integer number $N_{0}=\left[I_{p} / \omega\right]+1$ of relatively low fundamental frequency $\omega \ll I_{p}$ required to overcome the ionization threshold (here $I_{p}$ is the ionization potential of a laser-irradiated system and $[x]$ denotes an integer part of variable $x$; the atomic system of units is used unless stated otherwise), as well as due to various related applications in mind $[5,7]$.

The $a b$ initio theoretical treatment of strong-field ionization is a very complex mathematical problem even for oneelectron (hydrogenlike) atoms since the two relevant interactions (viz. the long-range Coulomb interaction of active (optical) electron(s) with residual parent core and EM coupling to the laser field) are of comparable strength $[8,9]$. Nevertheless, in the last decade there was also an extensively growing interest in understanding the ionization behavior of molecules in strong fields, as lasers are currently being used for diverse applications, such as controlling the photofragmentation branching ratio of large molecules [10] or as "soft" ionizers for mass spectrometry [11]. In the very early experimental studies the ionization rates for molecules were found, in general, to have average magnitudes very similar to atoms if they have nearly identical binding energies [12]; 
however, further investigations have found some unexpected exceptions [13-16]. These later experiments showed that ionization is noticeably or strongly suppressed for $\mathrm{H}_{2}\left(\right.$ or $\left.\mathrm{D}_{2}\right)$ and $\mathrm{O}_{2}$ as compared to their companion atoms ( $\mathrm{Ar}$ and $\mathrm{Xe}$, respectively), whereas ionization rates for $\mathrm{N}_{2}$ and $\mathrm{F}_{2}$ are comparable to their atomic counterpart Ar under the same laser pulses.

Although $a b$ initio calculations for the ionization rates of atoms are readily available, at least within the single-activeelectron (SAE) approximation, this is not the case for molecules. Unfortunately, quantum calculations on even relatively simple diatomic molecules are extremely difficult, therefore, strong-field molecular ionization is primarily treated nowadays, using the SAE-based general strong-field approaches, viz. either tunneling theory $[17,18]$ and/or conventional strong-field approximation (SFA) [8] with the related particular Keldysh-Faisal-Reiss (KFR) theories [19]. The ionization rates for molecules can be also calculated, in principle, based on various pure numerical procedures and methods, such as the time-dependent density-functional theory (TD-DFT) [20] including many-electron effects; however, the related results seem to be very computationally demanding and hardly available for reliable and transparent interpretation. When considering the ionization of molecules versus atoms, effects due to the additional degrees of freedom in molecules should also be evaluated because the ionization rate of molecules can further be affected by their rotational and vibrational motion. Also, the electronic cloud of an atom is mostly spherically symmetric, whereas for molecules it is not; this may particularly affect the produced molecular photoelectron angular distributions (PADs), which are expected to have noticeable differences from respective atomiclike ones generally dominated along the incident field polarization. The recent investigation [21] of the influence of vibrational motion and field-induced changes in bond length on the ionization rates of $\mathrm{H}_{2}$ and $\mathrm{O}_{2}$ did predict some reduced ionization yields relative to companion atoms of the same ionization potential; however, this reduction was verified to be too small and quite insufficient to explain the observed suppression. The other recently developed SAEbased strong-field models of molecular ionization-the socalled MO-SFA model [22] and alternative AmmosovDelone-Krainov (ADK)-based models (e.g., [23] and the socalled MO-ADK model [24])—were able to provide one with a more clear insight and interpretation of the origin of enhanced (or suppressed) ionization in diatomic molecules with respect to their atomic counterparts. Particularly, the MO-SFA model [22] (based, in fact, on the standard KFR approach) succeeded in quantitative description of molecular strong-field ionization of $\mathrm{N}_{2}$ and $\mathrm{O}_{2}$ in terms of atomic photoionization rates modified by constructive (or destructive) interference of ionization from two atomic centers separated by the internuclear distance $R_{0}$.

In the meantime, the latter main idea about the interference of atomic photoionization amplitudes obviously goes back to the work of Cohen and Fano [25], where the photoionization of $\mathrm{H}_{2}, \mathrm{~N}_{2}$, and $\mathrm{O}_{2}$ was considered within the frame of the first order of the Born approximation with respect to EM interaction with an incident far-ultraviolet radiation field. Owing to the mentioned interference is highly destructive for diatomics having an outermost valence shell [corresponding to the highest occupied molecular orbital (HOMO)] of antibonding symmetry ( such as $1 \pi_{g}$ in $\mathrm{O}_{2}$ ), this was identified in [22] as the reason of a high suppression in respective molecular ionization rates. Accordingly, for diatomics having the HOMO of bonding symmetry (such as $3 \sigma_{g}$ in $\mathrm{N}_{2}$ ), this interference is always constructive and, therefore, there was no suppression found in respective molecular ionization rates. On the other hand, the explanation of suppressed and/or enhanced molecular ionization in laser-irradiated diatomics is not so straightforward within the ADK-based approach [24]. The related MO-ADK model seems to be rather sophisticated because, besides the conventional tunneling theory, it is also based on the implement of the so-called multiple scattering method quite artificially invoked for proper finding of additional (fitting) parameters contained in preexponential factors of respective derived tunneling molecular ionization rates. Moreover, the validity of MO-ADK model is restricted to only tunneling regime of ionization (i.e., either very high-intensity or low-frequency laser field, for which the value of Keldysh parameter $\gamma \ll 1$ ). At last, unlike the MO-SFA approach, in which the initial molecular state (viz. HOMO) is considered as substantially twocentered (though, an approximate) MO; in MO-ADK theory the HOMO is always approximated by only one onecentered AO. This particularly means that, in contrast to the MO-SFA approach, the MO-ADK theory developed in [24] is not suitable for adequate description of related twocentered interference phenomena in molecular photoelectron and high-harmonic spectra (see, e.g., [22,26]). In addition, since the form of ADK formula used in [24] implies that ionization may occur only along (or opposite) the polarization of incident laser field, the respective developed MOADK model is not directly applicable for calculation of the respective angular differential molecular ionization rate (i.e., PAD). To conclude, the two aforementioned different strongfield approaches under discussion also predicted a high suppression for ionization in diatomic molecule $\mathrm{F}_{2}$ (that, like $\mathrm{O}_{2}$, is known to have antibonding $\pi_{g}$ outermost valence shell) with respect to ionization of its companion species $\mathrm{N}_{2}$ and $\mathrm{Ar}$ of nearly identical binding energies, contrary to the relevant results of later recent experiment [16]. Furthermore, the quite ad hoc interpretation of high suppression in strong-field ionization of a $\mathrm{D}_{2}$ molecule (compared to $\mathrm{Ar}$ ) proposed in terms of the MO-ADK theory seems to be inconsistent with respective model-related results of MO-ADK calculations (see, e.g., Fig. 6 presented in [24]), which, contrary to relevant results of recent experiments $[13,16]$, rather demonstrate no high suppression in ionization of $\mathrm{D}_{2}$ in the strong-field domain (i.e., only where the tunneling theory is generally valid).

The alternative calculations of strong-field ionization were also recently performed in [27] to inspect the results of $[22,24]$ related to a particular $\mathrm{N}_{2}$ molecule within the framework of the two different general strong-field approaches under discussion - the MO-SFA (both for the "length" and "velocity" gauge for the Hamiltonian of EM interaction with incident laser field) and, separately, MO-ADK (although, without any implement of the multiple-scattering method). Particularly, the orientation behavior of $\mathrm{N}_{2}$ ionization rates 
(depending on the direction of internuclear axis orientation with respect to the laser field polarization) was found to be quite a different if calculated in the velocity-gauge version of the MO-SFA approach compared to that suggested by the length-gauge and/or MO-ADK theory. In this regard it is worth noting a counterintuitive conclusion made in [27] that within the velocity gauge the ionization $\mathrm{N}_{2}$ predominates when the molecule is aligned perpendicular to the incident laser field polarization (see Fig. 4 in [27]). The latter conclusion seems to be quite a questionable and hardly can be accepted as correct since it is obviously based on some sad inaccuracy in related numerical calculations of generalized Bessel functions as well as the angular dependence of the Fourier transform of initial molecular state in $\mathrm{N}_{2}$ (i.e., $3 \sigma_{g}$ outermost valence shell, see also Sec. III for details and relevant discussion therein).

To summarize, the current state of the art in strong-field molecular ionization theory seems to be still far from a sufficiently clear insight due to numerous controversial results obtained within different approaches and methods that, thus, need some revising and/or improving. In the meantime, the KFR-based theory of strong-field ionization is recognized as a more general and rigorous theory as compared to any ADK-theory (the latter, in fact, can be derived from a general SFA-based $S$-matrix formalism for a particular case of too strong and/or low-frequency incident field, see, e.g., [28]). This all gave us a strong motivation to undertake one more attempt to consider the strong-field ionization of laserirradiated light homonuclear diatomics in terms of a conventional nonrelativistic SFA-approach similar to that previously proposed in [22]. Unlike [22], the strong-field molecular ionization model proposed in the present paper is entirely SAEbased as it implies analytical representation for Fourier transform of one-electron (though, also two-centered) wave function of initial molecular state by means of consequent applying the standard linear combination of atomic orbitals (LCAO) and molecular orbitals (MO) method. On the other hand, unlike the MO-ADK theory, the presently proposed SFA-LCAO model implies that photoelectron emission occurs along any arbitrary spatial direction with respect to the internuclear axis and/or incident field polarization. The latter allows, particularly, for direct calculation and adequate interpretation the produced molecular PADs that might provide one with additional useful information about some individual features inherent to the spatial configuration of the molecular valence shell under ionization, such as distribution of respective electronic density, which generally is spherically asymmetric. At last, the proposed SFA-LCAO model is not restricted to a consideration of ionization from only one, single MO (of the smallest binding energy) corresponding to the HOMO, but the comparable contributions from others (inner) molecular orbitals (closest to the HOMO, although, of larger binding energies and different bonding symmetry, spatial configuration, and number of valence electrons) can be additionally and equally well incorporated. The related results for calculated total ionization rates (or produced photoion yields) are fairly well consistent with available experimental data and able to provide one with a transparent physical interpretation of the nature and origin of high suppression or no suppression in molecular ionization in $\mathrm{O}_{2}$ and
$\mathrm{N}_{2}$ compared to their companion atoms or other diatomics revealed in relevant experiments. As to the case of $F_{2}$, it is a very special one, as the $X^{1} \Sigma_{g}^{+}$ground state of $\mathrm{F}_{2}$ is known as a singlet [16], so that its outermost $1 \pi_{g}$ valence shell is closed and, thus, differs from the opened outermost $1 \pi_{g}$ valence shell in $\mathrm{O}_{2}$. Moreover, unlike $\mathrm{F}_{2}$, the triplet ground state $X^{3} \Sigma_{g}^{-}$in $\mathrm{O}_{2}$ is of odd parity with respect to the reflection of electrons in the plane, which passes perpendicularly through the middle of the internuclear molecular axis. Thus, the initial two-centered molecular wave function corresponding to the outermost $1 \pi_{g}$ valence shell in $\mathrm{F}_{2}$ cannot be adequately reproduced yet in the same way as $1 \pi_{g} \mathrm{MO}$ in $\mathrm{O}_{2}$ (i.e., just as an antibonding superposition of two one-electron $2 p$ AOs), but requires a more careful (e.g., at least, twoelectron) consideration quite different from SAE approximation currently applied. In our opinion, the latter-mentioned difference seems to be also the most probable reason, for which the $\mathrm{F}_{2}$ related results of recent experiments (e.g., [16]) are not consistent with predictions of MO-SFA [22] and MOADK [24] models, since the latter treatment should substantially incorporate many-electron effects taken into consequent account under the ionization process; that is, however, beyond the scope of our present consideration.

\section{BACKGROUND THEORY OF STRONG-FIELD IONIZATION MODEL: BASIC ASSUMPTIONS AND ANALYTICAL RELATIONS}

According to the above-mentioned KFR theories [19], the time-independent strong-field amplitude of multiphoton ionization can be particularly represented as the standard $S$-matrix element of the EM interaction $\hat{W}(\mathbf{r}, t)$ with incident laser field

$$
F_{i \rightarrow f}^{(\mathrm{ATI})}(\mathbf{p}) \approx-i \int_{-\infty}^{\infty} d t\left\langle\psi_{\mathbf{p}}(\mathbf{r}, t)|\hat{W}(\mathbf{r}, t)| \Phi_{n}(\mathbf{r}, t)\right\rangle,
$$

i.e., via the $S$ matrix of EM transition of unperturbed laserirradiated system (with no incident EM laser field present) from the respective initial discrete state $\Phi_{n}(\mathbf{r}, t)=\Phi_{n}(\mathbf{r}) \exp ($ $-i \varepsilon_{n}^{(0)} t$ ) (here $\varepsilon_{n}^{(0)}=-I_{p}^{(n)}$ is the binding energy of the $n$th discrete level) to the field-perturbed continuum states $\psi_{\mathbf{p}}(\mathbf{r}, t)$ corresponding to motion of a free electron with a definite value of canonical momentum $\mathbf{p}$ driven in continuum by the incident laser field only. The latter continuum wave function $\psi_{\mathbf{p}}(\mathbf{r}, t)$ is the nonrelativistic Volkov's wave function (see, e.g., $[8,29])$ or just the exact solution of the time-dependent Schrödinger equation with the Hamiltonian $\hat{H}_{F}=\hat{\mathbf{p}}^{2} / 2$ $+\hat{W}(\mathbf{r}, t)$ (here $\hat{\mathbf{p}}=-i \nabla$ is the operator of electron canonical momentum). Thus, throughout our present consideration the SAE approximation is further supposed to be always applicable to the laser-irradiated system under ionization, so that only one (the so-called active or optical) electron (moving in some effective SAE binding potential $V(\mathbf{r})$ of the parent core averaged over the time-independent charge distribution of all remaining inactive electrons) is allowed to respond to the incident laser field. Moreover, we also follow the general concepts of the conventional SFA approach [8], according to 
which at any time moment of evolution the laser-irradiated system is considered as being under action of only one strong interactions of comparable strength [i.e., either EM interaction $W(\mathbf{r}, t)$ or binding potential $V(\mathbf{r})]$ separately, but never under two, simultaneously. Under this conventional supposition, the related photoionization amplitude (1) is also a kind of standard expression corresponding to the Keldysh amplitude of strong-field direct ATI process derived within frames of a more general SFA-based $S$-matrix formalism $[5,7,8]$. As a result, the related strong-field ionization amplitude (1) accounts for the so-called direct ATI process only and neglects the other strong-field ionization process-highorder (or rescattering) ATI, although, of considerably less probability, but responsible for the origin of a high-energy plateau in photoelectron spectra (see, also $[2,5,7,30]$, for more references and details). In the meantime, the SFAbased strong-field ionization amplitude (1) is known to be valid mostly within the photoelectron energy domain quite away from the ionization threshold $\left(\varepsilon_{\mathbf{p}}=p^{2} / 2 \gg \omega\right)$, where the influence of interaction with the parent residual core is generally negligibly small. This seems to be sufficiently well justified owing to a relatively high photoelectron energy $U_{p}$ $\gg \omega$ corresponding to a very large number $N>N_{0} \gg 1$ of incident photons absorbed in the strong-field multiphoton ionization process under consideration.

Under conditions of a nonrelativistic treatment of the problem, the photoelectron energy $\varepsilon_{\mathbf{p}}=\mathbf{p}^{2} / 2$ and ponderomotive energy $U_{p}$ (the energy of oscillating motion of a free electron driven by incident laser field) are both supposed to be negligibly small as compared to the electron rest energy $\left(\varepsilon_{\mathbf{p}}, U_{p} \ll c_{0}^{2}\right.$, where $c_{0} \approx 137$ is the light velocity in vacuum). This particularly means that, to fairly good accuracy, the EM interaction of an active (optical) electron with a strong driving laser field can be considered within the dipole ( or longwavelength) approximation (neglecting any photon momenta, $\mathbf{k}=0$ ) wherein the incident field strength $\mathbf{E}(t)$ and associated vector potential $\mathbf{A}(t)$ are independent on coordinate radius vector $\mathbf{r}$, but both are functions only of time $t$. Hence, the respective Hamiltonian of EM interaction of an electron with incident laser field may be written, for example, in (A·p) (the so-called velocity gauge) form

$$
\hat{W}(\mathbf{r}, t)=\frac{1}{c_{0}} \mathbf{A}(t) \cdot \hat{\mathbf{p}}+\frac{1}{2 c_{0}^{2}} \mathbf{A}^{2}(t) .
$$

Then, the exact solution of time-dependent Schrödinger equation corresponding to the EM interaction (2) reads as (e.g., $[8,29])$

$$
\psi_{\mathbf{p}}(\mathbf{r}, t)=(2 \pi)^{-3 / 2} \exp \left[i \mathbf{p} \cdot \mathbf{r}-\frac{i}{2} \int_{-\infty}^{t}\left(\mathbf{p}+\frac{1}{c_{0}} \mathbf{A}\left(t^{\prime}\right)\right)^{2} d t^{\prime}\right] .
$$

Owing to a very high intensity the incident laser field can be described entirely classically, so that its vector potential $\mathbf{A}(t)$ is given by the following conventional form:

$$
\mathbf{A}(t)=\left(c_{0} / \omega\right) \mathbf{e} E \cos (\omega t),
$$

where $\mathbf{e}$ and $E$ are the unit polarization vector and electric vector strength of incident EM field, respectively. For the latter particular case of linearly polarized monochromatic incident field (4) under further consideration, the explicit expression for the nonrelativistic Volkov's wave function (3) can be also represented in the form of expansion in harmonics of laser field frequency $\omega[8,19]$

$$
\psi_{\mathbf{p}}(\mathbf{r}, t)=|\mathbf{p}\rangle \sum_{s=-\infty}^{\infty} B_{s}\left(\zeta(\mathbf{p}) ; \frac{\eta}{2}\right) \exp \left[-i\left(\frac{\mathbf{p}^{2}}{2}+U_{p}+s \omega\right) t\right],
$$

i.e., in terms of electron plane wave functions $|\mathbf{p}\rangle$ and the generalized Bessel function of the first kind and sth order

$$
B_{s}(x ; y)=\sum_{k=-\infty}^{\infty} J_{s-2 k}(x) J_{k}(y),
$$

and of two real arguments, where $J_{s}(x)$ is an ordinary Bessel function of the first kind and sth order of real argument $x$, moreover, the two dimensionless parameters $\zeta(\mathbf{p})$ $=(\mathbf{E} \cdot \mathbf{p}) / \omega^{2}=\left(E p / \omega^{2}\right) \cos \theta$ and $\eta=U_{p} / \omega=E^{2} /\left(4 \omega^{3}\right)$ (the latter is also known as the so-called Reiss parameter [8]) have been additionally introduced here.

By means of direct substitution the explicit expression for Volkov's wave function (5) to Eq. (1) and performing an elementary analytical integration over time variable (that just results in the singular energy-dependent Dirac $\delta$ function expressing the conservation of total energy in the process under consideration), one can derive the amplitude of direct ATI process in the following explicit form:

$$
F_{i \rightarrow f}^{(n)}(\mathbf{p}) \approx 2 \pi \sum_{N=-\infty}^{\infty} f_{N}^{(n)}(\mathbf{p}, \eta) \delta\left(\varepsilon_{\mathbf{p}}^{(n)}+I_{p}^{(n)}+U_{p}-N \omega\right) .
$$

Here each $N$ th item of summation is the well-known expression for Keldysh partial ( $N$-photon) amplitude of strong-field ionization (see also, e.g., $[19,29]$ )

$$
f_{N}^{(n)}(\mathbf{p}, \eta)=-i\left(U_{p}-N \omega\right) B_{-N}\left(\zeta(\mathbf{p}) ; \frac{\eta}{2}\right) \sqrt{N_{e}^{(n)}} \Phi_{n}(\mathbf{p})
$$

because of the direct ATI process along the course of which the field-irradiated system with a total number $N_{e}^{(n)}$ of identical electrons in $n$th initial state absorbs a net number $N$ of incident photons, whereas

$$
\Phi_{n}(\mathbf{p})=\left\langle\mathbf{p} \mid \Phi_{n}(\mathbf{r})\right\rangle=(2 \pi)^{-3 / 2} \int d \mathbf{r} \exp (-i \mathbf{p} \cdot \mathbf{r}) \Phi_{n}(\mathbf{r})
$$

is just the Fourier transform of the wave function $\Phi_{n}(\mathbf{r})$ of initial (unperturbed by incident laser field) $n$th discrete state of laser-irradiated system under ionization. Thus, the presence of singular Dirac $\delta$ function on the right-hand side of (7) makes the final energy $\varepsilon_{\mathbf{p}}$ of emitted ATI photoelectron take no arbitrary values, but only discrete ones $\varepsilon_{N, \mathbf{p}}^{(n)}$ determined by

$$
\varepsilon_{N, \mathbf{p}}^{(n)}=\mathbf{p}_{N}^{2} / 2=N \omega-I_{p}^{(n)}-U_{p}
$$

and separated from each other by the incident laser field fundamental frequency $\omega$. Consequently, within the currently 
applied approach, the produced photoelectron spectrum is always represented by a set of discrete peaks of energies $\varepsilon_{N, \mathbf{p}}^{(n)}$ and respective heights (intensities) determined by corresponding partial (of $N$ th order) ATI amplitudes (8). The respective differential ionization rates $w_{N}^{(n)}\left(\mathbf{p}_{N}\right)$ of $n$th valence shell (containing $N_{e}^{(n)}$ identical electrons) due to absorption of $N$ incident photons and emission of photoelectron of final momentum $p_{N}^{(n)}=\sqrt{2\left(N \omega-I_{p}^{(n)}-U_{p}\right)}$ to a fixed spatial direction along the solid angle element $d \mathrm{O}_{\mathbf{p} N}$ are conventionally found by means of standard procedure of a squaring module of total ATI amplitude (7) divided by a long normalization time and integrated over entire phase-space volume of emitted photoelectron final states

$$
\begin{aligned}
w_{N}^{(n)}\left(\mathbf{p}_{N}, \eta\right)= & \frac{d R_{N}^{(n)}\left(p_{N}, \eta\right)}{d \mathrm{O}_{\mathbf{p}_{N}}}=\frac{N_{e}^{(n)}}{(2 \pi)} p_{N}^{(n)}\left(U_{p}\right. \\
& -N \omega)^{2}\left|\Phi_{n}\left(\mathbf{p}_{N}^{(n)}\right)\right|^{2} B_{-N}^{2}\left(\zeta\left(\mathbf{p}_{N}^{(n)}\right) ; \frac{\eta}{2}\right) .
\end{aligned}
$$

The introduced $N$ th integral ionization rates $R_{N}^{(n)}\left(p_{N}, \eta\right)$ determine the respective integral photoelectron spectra, which are found by integration of partial differential ionization rates $w_{N}^{(n)}\left(\mathbf{p}_{N}^{(n)}, \eta\right)$ over all angles of photoelectron emission. The partial differential rates (11) determine the produced photoelectron spectra observed along the fixed direction of photoelectron emission (e.g., with respect to the direction of incident field polarization) as well as the respective PAD corresponding to angular distribution of certain $\left(N\right.$ th) photoelectron peak. The total PAD $P_{\mathrm{PAD}}^{(n)}(\mathbf{p}, \eta)$ produced by all emitted photoelectrons can be found directly from (11) by means of summation over all contributing ATI peaks of energies corresponding to fixed photoelectron energies $\varepsilon_{N, \mathbf{p}}^{(n)}$ in final continuum states (or, so-called open direct ATI channels)

$$
\begin{aligned}
P_{\mathrm{PAD}}^{(n)}(\mathbf{p}, \eta)= & \frac{N_{e}^{(n)}}{(2 \pi)} \sum_{N \geqslant N_{0}^{(n)}} p_{N}^{(n)}\left(U_{p}-N \omega\right)^{2} B_{-N}^{2}\left(\zeta\left(\mathbf{p}_{N}^{(n)}\right) ; \frac{\eta}{2}\right) \\
& \times\left|\Phi_{n}\left(\mathbf{p}_{N}^{(n)}\right)\right|^{2}
\end{aligned}
$$

where $N_{0}^{(n)}=\left[\left(I_{p}^{(n)}+U_{p}\right) / \omega\right]+1([x]$ denotes an integer part of variable $x$ ) is the minimum number of absorbed incident photons required for ionization of laser-irradiated system. Thus, due to a presence of a strong incident laser field, the ionization threshold is raised to a corresponding value of ponderomotive energy $U_{p}$ also contained in singular Dirac $\delta$ function of amplitude (7), so that the latter minimum number $N_{0}^{(n)}$ $=N_{0}^{(n)}(\eta)$ of absorbed photons is strongly dependent on incident field intensity. Analogously, the total ionization rate $\Gamma_{\text {ion }}^{(n)}(\eta)$ (or, in other words, the total rate of photoelectron and/or ion yield) from $n$th initial state can be found by means of integration of partial differential rates (11) over all angles of photoelectron emission and, moreover, summation over all contributing ATI channels (or number $N$ of incident photons absorbed)

$$
\begin{aligned}
\Gamma_{\text {ion }}^{(n)}(\eta)= & \sum_{N \geqslant N_{0}^{(n)}(\eta)} \int w_{N}^{(n)}\left(\mathbf{p}_{N}^{(n)}, \eta\right) d \mathrm{O}_{\mathbf{p}_{N}}=\frac{N_{e}^{(n)}}{(2 \pi)} \sum_{N \geqslant N_{0}^{(n)}(\eta)} p_{N}^{(n)} \\
& \times\left(U_{p}-N \omega\right)^{2} \int d \mathrm{O}_{\mathbf{p}_{N} B_{-N}^{2}}\left(\zeta\left(\mathbf{p}_{N}^{(n)}\right) ; \frac{\eta}{2}\right)\left|\Phi_{n}\left(\mathbf{p}_{N}^{(n)}\right)\right|^{2} .
\end{aligned}
$$

\section{SAE EXTENSION TO STRONG-FIELD MOLECULAR IONIZATION: THE GENERALIZED SFA-LCAO MODEL}

The expressions (11)-(13) are also the main basis and starting point of other similar KFR-based strong-field atomic and molecular ionization models developed earlier (e.g., $[22,23])$. Therefore, let us outline now the main distinctive features differentiating the proposed molecular strong-field ionization model from any other ones mentioned above. According to currently used version of SFA, all the information about specified properties of laser-exposed system under this SAE-based consideration is contained in the form of the ionization potential $I_{p}^{(n)}$ and Fourier transform $\Phi_{n}(\mathbf{p})$ $=\left\langle\mathbf{p} \mid \Phi_{n}(\mathbf{r})\right\rangle$ of approximate one-electron wave function $\Phi_{n}(\mathbf{r})$ of the initial discrete state that is supposed to be undistorted by incident laser field. For the particular case of laser-irradiated homonuclear diatomic molecules under consideration, these functions correspond to a separate ( $n$ th) valence shell under ionization and are further used in explicit analytical form derived within the linear combination of atomic orbitals and molecular orbitals (LCAO-MO) method, which is also known as a very effective and powerful tool for various molecular calculations. Particularly, according to standard LCAO-MO method, the molecular wave function of $n$th molecular valence shell is approximately considered as entirely SAE two-centered MO, which is chosen as a linear superposition of predominantly contributing (jth) onecentered AOs $\phi_{j}^{(n)}(\mathbf{r})$

$$
\begin{aligned}
\Phi_{n}\left(\mathbf{r}_{1} ; \mathbf{r}_{2}\right)= & \Phi_{n}\left(\mathbf{r}+\mathbf{R}_{0} / 2 ; \mathbf{r}-\mathbf{R}_{0} / 2\right) \\
= & \sum_{j}\left\{\sqrt{2\left[1 \pm S_{j}^{(n)}\left(R_{0}\right)\right]}\right\}^{-1}\left[\phi_{j}^{(n)}\left(\mathbf{r}+\mathbf{R}_{0} / 2\right) \pm \phi_{j}^{(n)}(\mathbf{r}\right. \\
& \left.\left.-\mathbf{R}_{0} / 2\right)\right]
\end{aligned}
$$

separated by internuclear distance $R_{0}$. Here

$$
S_{j}^{(n)}\left(R_{0}\right)=\int d \mathbf{r} \phi_{j}^{(n)}\left(\mathbf{r}+\mathbf{R}_{0} / 2\right) \phi_{j}^{(n)}\left(\mathbf{r}-\mathbf{R}_{0} / 2\right)
$$

is the respective atomic orbital overlap integral and the minus sign on the right-hand side of (16) corresponds to an antibonding valence shell (e.g., $\sigma_{u}$ or $\pi_{g}$ ), whereas the plus sign corresponds to a bonding valence shell (e.g., $\sigma_{g}$ or $\pi_{u}$ ). Let us also recall that a symmetric superposition of contributing AOs does not necessarily imply the so-called gerade (e.g., $\sigma_{g}$ ) MO only, but it is also inherent to ungerade (e.g., $\pi_{u}$ ) bonding MO, as well as an antisymmetric superposition may correspond to both gerade (e.g., $\pi_{g}$ ) antibonding MO and ungerade (e.g., $\sigma_{u}$ ) antibonding $\mathrm{MO}$. 
The choice (14) does provide a negligibly small electron density near the central region between the atomic nuclei in homonuclear diatomic for antibonding symmetry of respective molecular valence shell (e.g., $1 \pi_{g} \mathrm{MO}$ in $\mathrm{O}_{2}$ ) as well as a predominant concentration of electron density in the internuclear region for the case of bonding symmetry (e.g., for $3 \sigma_{g} \mathrm{MO}$ in $\mathrm{N}_{2}$ ). But, contrary to the unambiguous statement made in Ref. [22], only such a choice itself is still quite insufficient to provide also a negligibly small electron density along the internuclear molecular axis inherent to, for example, $\pi$ valence shells (viz., antibonding $\pi_{g}$ and bonding $\pi_{u}$ ). Meantime, the spatial orientation of $\pi$ valence shells is known as being quite different from the internuclear axis and, thus, with a negligibly small electron density along the internuclear molecular axis even for bonding $\pi_{u}$ valence shell, i.e., irrespective of bonding or antibonding symmetry only taken into consideration in Ref. [22]. The above choice (14) cannot provide a considerable electron density near the internuclear axis region inherent, for example, to $\sigma$ valence shells (viz., bonding $\sigma_{g}$ and antibonding $\sigma_{u} \mathrm{MO}$ ). However, the mentioned feature inherent to spatial configuration of $\sigma$ and $\pi$ valence shells can be fairly well reproduced with an appropriate choice of predominantly contributing AOs $\phi_{j}^{(n)}$ $\times(\mathbf{r})$, which have the required spatial configuration and adequately (although, approximately) reproduce the spatial distribution of electronic density with respect to the internuclear axis in respective $n$th MOs under consideration. Particularly, the approximate two-centered one-electron molecular wave function for bonding $2 p \pi_{u}\left(1 \pi_{u}\right)$ and/or antibonding $2 p \pi_{g}\left(1 \pi_{g}\right)$ valence shells (e.g., in $\mathrm{O}_{2}$ ) can be composed from symmetric (for $1 \pi_{u}$ ) and/or antisymmetric (for $1 \pi_{g}$ ) superposition of one-electron hydrogenlike $2 p$ AOs respectively, e.g., from either $2 p_{x}$ or $2 p_{y}$ orbitals

$$
\begin{aligned}
& \phi_{2 p_{x}}^{\left(1 \pi_{u}\right)}(\mathbf{r})=\left(\frac{Z_{\mathrm{eff}}^{(2)}}{a_{2}}\right)^{5 / 2} \frac{r}{\sqrt{\pi}} \exp \left(-\frac{Z_{\mathrm{eff}}^{(2)}}{a_{2}} r\right) \sin \left(\theta_{R}\right) \cos \left(\varphi_{R}\right) \\
& \phi_{2 p_{y}}^{\left(1 \pi_{g}\right)}(\mathbf{r})=\left(\frac{Z_{\mathrm{eff}}^{(2)}}{a_{2}}\right)^{5 / 2} \frac{r}{\sqrt{\pi}} \exp \left(-\frac{Z_{\mathrm{eff}}^{(2)}}{a_{2}} r\right) \sin \left(\theta_{R}\right) \sin \left(\varphi_{R}\right),
\end{aligned}
$$

which two, such as $\pi$ molecular shells, are predominantly aligned along a direction perpendicular to the internuclear molecular axis. Here $\theta_{R}$ and $\varphi_{R}$ are the polar and azimuthal angles with respect to the $O Z$-coordinate axis (which is supposed to be coincident with the internuclear axis), while $a_{j}$ $=j a_{0}$ is the $j$ th Bohr orbital radius and $Z_{\text {eff }}^{(n)}$ is the effective charge corresponding to the molecular binding energy $\varepsilon_{0}^{(n)}=$ $-\kappa_{n}^{2} / 2=-I_{p}^{(n)}=-\left(Z_{\text {eff }}^{(n)} / a_{j}\right)^{2} / 2$ of $n$th valence shell under consideration.

Analogously, the approximate two-centered molecular wave functions of bonding $2 p \sigma_{g}\left(3 \sigma_{g}\right)$ and/or antibonding $2 s \sigma_{u}\left(2 \sigma_{u}\right)$ valence shells (e.g., in $\left.\mathrm{N}_{2}\right)$ can be composed, for example, from symmetric (for $3 \sigma_{g}$ ) and/or antisymmetric (for $2 \sigma_{u}$ ) superposition of hydrogenlike $2 p_{z}$ (for $3 \sigma_{g}$ ) or $2 s$ (for $2 \sigma_{u}$ ) orbitals, respectively

$$
\begin{gathered}
\phi_{2 p_{z}}^{\left(3 \sigma_{g}\right)}(\mathbf{r})=\left(\frac{Z_{\mathrm{eff}}^{(2)}}{a_{2}}\right)^{5 / 2} \frac{r}{\sqrt{\pi}} \exp \left(-\frac{Z_{\mathrm{eff}}^{(2)}}{a_{2}} r\right) \cos \left(\theta_{R}\right) \\
\phi_{2 s}^{\left(2 \sigma_{u}\right)}(\mathbf{r})=\frac{1}{\sqrt{\pi}}\left(\frac{Z_{\mathrm{eff}}^{(2)}}{a_{2}}\right)^{5 / 2}\left(1-\frac{Z_{\mathrm{eff}}^{(2)}}{a_{2}} r\right) \exp \left(-\frac{Z_{\mathrm{eff}}^{(2)}}{a_{2}} r\right),
\end{gathered}
$$

the first of which is known as prolate along the internuclear molecular axis. One can directly examine that predominantly contributing AOs chosen in the form (16)-(19) are quite appropriate to a fairly well approximate reproducing the spatial distribution of respective molecular electronic density. The latter molecular electronic density for $\sigma$ molecular valence shells is known as considerably prevailing near (or extended along) the internuclear axis, whereas, for $\pi$ valence shells, it is a negligibly small nearly around the same space region. The effective charge $Z_{\text {eff }}^{(n)}$ corresponding to effective longrange Coulomb binding potential of the residual molecular ion can be found owing to the correct (calculated or experimental) value of molecular binding energy $\varepsilon_{0}^{(n)}$ for respective molecular valence shell. The latter binding energies are supposed to be already known for separate molecular valence shells under consideration, particularly, $I_{p}^{\left(1 \sigma_{g}\right)} \approx 15.43 \mathrm{eV}$ for diatomic $\mathrm{H}_{2}$, whereas, $I_{p}^{\left(3 \sigma_{g}\right)} \approx 15.58 \mathrm{eV}, I_{p}^{\left(1 \pi_{u}\right)} \approx 16.96 \mathrm{eV}$, and $I_{p}^{\left(2 \sigma_{u}\right)} \approx 18.73 \mathrm{eV}$ for the three highest molecular valence shells in diatomic $\mathrm{N}_{2}$, or $I_{p}^{\left(1 \pi_{g}\right)} \approx 12.07 \mathrm{eV}, I_{p}^{\left(1 \pi_{u}\right)} \approx 16.26 \mathrm{eV}$, and $I_{p}^{\left(3 \sigma_{g}\right)} \approx 18.18 \mathrm{eV}$ for the three highest molecular valence shells in diatomic $\mathrm{O}_{2}$.

The explicit expressions (16)-(19) also allow for analytical calculation of matrix elements (9) corresponding to the Fourier transform of respective molecular valence shell represented by approximate two-centered MO under ionization. Particularly, for bonding $3 \sigma_{g}$ and antibonding $2 \sigma_{u}$ molecular valence shells one can derive

$$
\Phi_{3 \sigma_{g}}\left(\mathbf{p}_{N}, \mathbf{R}_{0}\right)=\frac{i C\left(\kappa_{n}\right) 2^{5} \kappa_{n}^{7 / 2} p_{N} \cos \left(\theta_{\mathbf{p}}\right)}{\pi \sqrt{2}\left(p_{N}^{2}+\kappa_{n}^{2}\right)^{3}} \frac{\cos \left[\left(\mathbf{p}_{N} \cdot \mathbf{R}_{0}\right) / 2\right]}{\sqrt{2\left[1+S_{2 p_{z}}\left(R_{0}\right)\right]}}
$$

and

$$
\Phi_{2 \sigma_{u}}\left(\mathbf{p}_{N}, \mathbf{R}_{0}\right)=\frac{C\left(\kappa_{n}\right) 2^{4} \kappa_{n}^{5 / 2}\left(\kappa_{n}^{2}-p_{N}^{2}\right)}{\pi \sqrt{2}\left(p_{N}^{2}+\kappa_{n}^{2}\right)^{3}} \frac{\sin \left[\left(\mathbf{p}_{N} \cdot \mathbf{R}_{0}\right) / 2\right]}{\sqrt{2\left[1-S_{2 s}\left(R_{0}\right)\right]}},
$$

respectively.

Accordingly, for bonding $1 \pi_{u}$ and antibonding $1 \pi_{g}$ molecular valence shells

$$
\Phi_{1 \pi_{u}}\left(\mathbf{p}_{N}, \mathbf{R}_{0}\right)=\frac{i C\left(\kappa_{n}\right) 2^{5} p_{N} \kappa_{n}^{7 / 2} \sin \left(\theta_{\mathbf{p}}\right)}{\pi \sqrt{2}\left(p_{N}^{2}+\kappa_{n}^{2}\right)^{3}} \frac{\cos \left[\left(\mathbf{p}_{N} \cdot \mathbf{R}_{0}\right) / 2\right]}{\sqrt{2\left[1+S_{2 p_{x}}\left(R_{0}\right)\right]}}
$$




$$
\Phi_{1 \pi_{g}}\left(\mathbf{p}_{N}, \mathbf{R}_{0}\right)=\frac{i C\left(\kappa_{n}\right) 2^{5} p_{N} \kappa_{n}^{7 / 2} \sin \left(\theta_{\mathbf{p}}\right)}{\pi \sqrt{2}\left(p_{N}^{2}+\kappa_{n}^{2}\right)^{3}} \frac{\sin \left[\left(\mathbf{p}_{N} \cdot \mathbf{R}_{0}\right) / 2\right]}{\sqrt{2\left[1-S_{2 p_{x}}\left(R_{0}\right)\right]}} .
$$

Here $\kappa_{n}=Z_{\text {eff }}^{(n)} / a_{2}$ and $\theta_{\mathbf{p}}$ is the polar angle of photoelectron emission with respect to the internuclear molecular axis $\left[\cos \left(\theta_{\mathbf{p}}\right)=\left(\mathbf{p} \cdot \mathbf{R}_{0}\right) / p R_{0}\right]$. Moreover, because of the velocitygauge form of SFA currently applied, the correction factor $C\left(\kappa_{n}\right)=\left(2 \kappa_{n} I_{p}^{(n)} / E\right)^{\kappa_{n}^{-1}}$ is also introduced to matrix elements (20)-(23) to incorporate the long-range Coulomb electronmolecular ion interaction in the final Volkov state into account [22]. For atomic orbital overlap integrals one can also derive

$$
S_{2 p_{x}}\left(R_{0}\right)=\left[1+\kappa_{n} R_{0}+\frac{2}{5}\left(\kappa_{n} R_{0}\right)^{2}+\frac{1}{15}\left(\kappa_{n} R_{0}\right)^{3}\right] \exp \left(-\kappa_{n} R_{0}\right),
$$

$$
S_{2 s}\left(R_{0}\right)=\left[1+\kappa_{n} R_{0}+\frac{1}{3}\left(\kappa_{n} R_{0}\right)^{2}+\frac{1}{15}\left(\kappa_{n} R_{0}\right)^{4}\right] \exp \left(-\kappa_{n} R_{0}\right),
$$

$$
\begin{aligned}
S_{2 p_{z}}\left(R_{0}\right)= & {\left[1+\kappa_{n} R_{0}+\frac{1}{5}\left(\kappa_{n} R_{0}\right)^{2}-\frac{2}{15}\left(\kappa_{n} R_{0}\right)^{3}-\frac{1}{15}\left(\kappa_{n} R_{0}\right)^{4}\right] } \\
& \times \exp \left(-\kappa_{n} R_{0}\right) .
\end{aligned}
$$

Meantime, for laser-irradiated atomic counterparts of diatomics $\mathrm{N}_{2}$ (or $\mathrm{H}_{2}$ ) and $\mathrm{O}_{2}$, under particular consideration, such as $\mathrm{Ar}$ and $\mathrm{Xe}$ (with $3 p$ and $5 p$ outermost atomic valence shells, respectively), the initial atomic ground state can be also approximately reproduced by corresponding oneelectron hydrogenlike atomic orbitals. Then, for $3 p_{z}, 3 p_{x}$, and $3 p_{y}$ hydrogenlike AOs (e.g., in Ar) corresponding to different possible values of associated magnetic quantum number $m(0$ for $3 p_{z}$ and \pm 1 for $3 p_{x} \pm i 3 p_{y}$ ), the analytical expression for respective atomic Fourier transforms $\Phi_{3 p_{z}}^{\left(3 p_{x}, 3 p_{y}\right)}(\mathbf{p})$ has the form

$$
\Phi_{3 p_{z}}^{\left(3 p_{x} \pm 3 p_{y}\right)}\left(\mathbf{p}_{N}\right)=\frac{C\left(\kappa_{n}\right) i 2^{4} \sqrt{2} \kappa_{n}^{11 / 2} p_{N}}{\pi \sqrt{3}\left(p_{N}^{2}+\kappa_{n}^{2}\right)^{4}}\left(\begin{array}{c} 
\pm \sin (\theta) \exp ( \pm i \varphi) \\
\sqrt{2} \cos (\theta)
\end{array}\right) .
$$

Here the angles $\theta$ and $\varphi$ are the polar and azimuthal angles of photoelectron emission with respect to the incident field polarization $[\cos (\theta)=(\mathbf{p} \cdot \mathbf{e}) / p]$, so that the angular factor on the right-hand side of (27) corresponds to the case of either $3 p_{x} \pm i 3 p_{y}$ (the upper part) or $3 p_{z}$ (the lower part) AOs. The effective charge $Z_{\text {eff }}$ of Coulomb atomic binding potential $V(\mathbf{r})=-Z_{\text {eff }} / r$ is also found from the experimental value of atomic ionization potential $\left(I_{p} \approx 15.75 \mathrm{eV}\right.$ for $\mathrm{Ar}$ and $I_{p}$ $\approx 12.07 \mathrm{eV}$ for $\mathrm{Xe}$ ) of respective atomic binding energy $\varepsilon_{0}^{(n)}=-\kappa_{n}^{2} / 2=-\left(Z_{\text {eff }}^{(n)} / a_{3}\right)^{2} / 2$. Thus, the Fourier transform $\Phi_{3 p}(\mathbf{p})$ corresponding to ionization of initial $3 p$ atomic state in Ar can be obtained from the expression (27) after averaging overmagnetic quantum number $m$ corresponding to averaging over all contributing $3 p$ atomic orbitals from which the photoelectron emission is equally possible.
It is interesting that the angular dependence of the derived matrix element (20) clearly indicates that the $3 \sigma_{g}$ valence shell is highly resistant to ionization along the direction perpendicular to the internuclear molecular axis $\left(\cos \left(\theta_{\mathbf{p}}\right) \approx 0\right)$, so that photoelectron emission from $3 \sigma_{g}$ is dominated primarily along the internuclear molecular axis $\left(\cos \left(\theta_{\mathbf{p}}\right) \approx 1\right)$ [see also Fig. 1(a)]. On the other hand, because of the generalized Bessel function contained in the ionization rate, (11) is generally maximal around the maximum of the first argument $\zeta\left(\mathbf{p}_{N}\right) \sim \cos (\theta)$, and the EM coupling of photoelectron in continuum to incident laser field is maximally strong mostly for photoelectrons emitted along the incident laser field polarization $(\cos (\theta) \approx 1)$ [Figs. 2(a) and 2(b)]. This particularly also means that the ionization of $3 \sigma_{g}$ (e.g., in $\mathrm{N}_{2}$ ) is to be maximal if the internuclear axis is strongly aligned along the laser field polarization [see Fig. 3(a)], that is, however, in contradiction to quite an opposite angular behavior of the $\mathrm{N}_{2}$ ionization rate suggested in [27] based on the velocity gauge form of the MO-SFA ionization model [22]. According to the latter result the ionization of the $\mathrm{N}_{2}$ molecule is predominant when its internuclear axis is perpendicular to the incident laser field polarization. By direct comparison to our current results presented in Figs. 2(a) and 3(a)-3(c) (also firmly and independently confirmed by Prof. H. R. Reiss, who was specially asked and kindly agreed to throughly verify them), one can see that the reason for such a contradiction is in quite different angular behavior of both the Fourier transform of $3 \sigma_{g}$ valence shell and the generalized Bessel function calculated in [27] for the same problem parameters (see, e.g., Figs. 4(e) and 4(f) presented therein). Thus, contrary to the conclusion made in [27], the quite opposite counterintuitive orientation-dependent behavior suggested therein for ionization of $\mathrm{N}_{2}$ is not somehow related to the velocity gauge form of the applied MO-SFA model, but rather just the result of inaccurate numerical calculation.

\section{CALCULATION, NUMERICAL RESULTS, AND DISCUSSION}

Thus, within the framework of the currently applied SFALCAO molecular ionization model, the associated ionization rate, the produced (ATI) photoelectron spectrum, and respective PAD are represented in closed and compact analytical form quite available for related direct numerical calculations. According to (11)-(13), these numerical calculations imply an accurate numerical calculation of generalized Bessel function $B_{m}(x ; y)$ for real values of its arguments. The next important issue is directly related to the procedure of approximate numerical calculation of formally infinite sum over $N$ corresponding to contribution of $N$ th open ATI channel. However, because of a negligible contribution from open ATI channels corresponding to very large values of $N$, this summation can be cut from above by a finite number $N_{\max }$ to take into account only ATI channels predominantly contributing to the total ionization rates (13) and respective total PAD (12). Namely, the number $N_{C}=N_{\max }-N_{0}^{(n)}$ of predominantly contributing ATI channels can be easily evaluated owing to the particular asymptotic property of the generalized Bessel function (5) which, at fixed values of its arguments $x$ and $y$, 


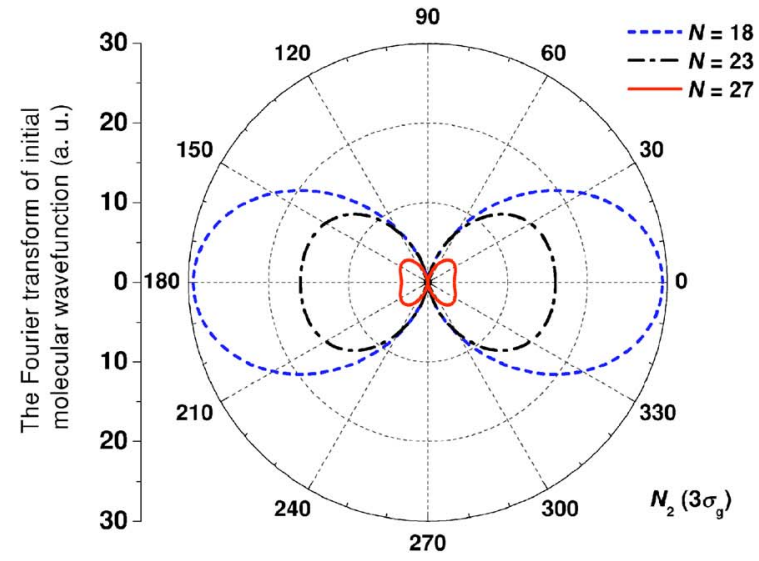

(a)

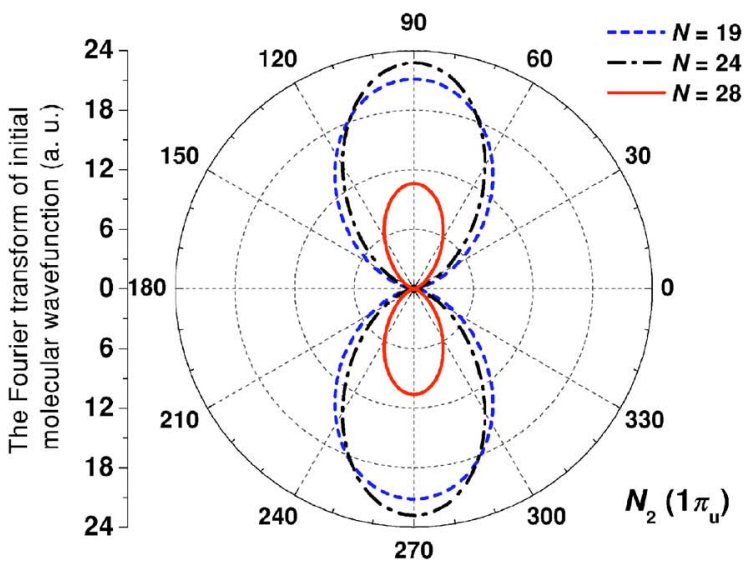

(b)

$\theta_{\mathrm{p}}$, Angle of Laser Field Polarization (degrees)

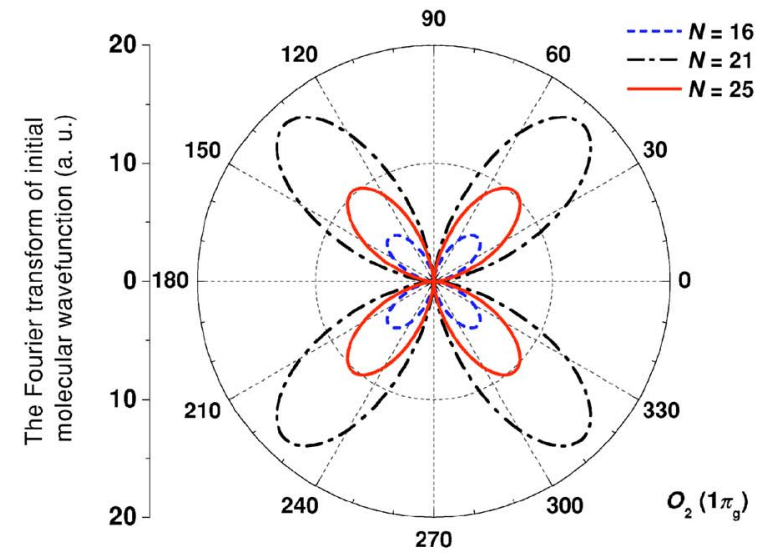

(c)

FIG. 1. The squared module $\left|\Phi\left(\mathbf{p}_{N}, \mathbf{R}_{0}\right)\right|^{2}$ of Fourier transforms (20)-(23) calculated for separate molecular valence shells in (a) (b) $\mathrm{N}_{2}$ and (c) $\mathrm{O}_{2}$ vs the angle $\theta_{\mathbf{p}}$ of photoelectron emission with respect to the internuclear molecular axis, which is supposed to be lined up in the horizontal direction. Those angular dependencies are all presented for only photoelectrons emitted along the laser field polarization $(\theta=0)$ of Ti:sapphire laser field $(\lambda \approx 800 \mathrm{~nm}, \hbar \omega$ $=1.6 \mathrm{eV})$ of fixed intensity $\left(I=2 \times 10^{14} \mathrm{~W} / \mathrm{cm}^{2}\right)$ and produced because of the absorption of the different number $N$ of laser photons beginning from minimum one $N_{0}$ required for ionization of respective separate valence shell: (a) $N_{0}=18$ for $3 \sigma_{g}$ in $N_{2}$, (b) $N_{0}=19$ for $1 \pi_{u}$ in $\mathrm{N}_{2}$, and (c) $N_{0}=16$ for $1 \pi_{g}$ in $\mathrm{O}_{2}$.

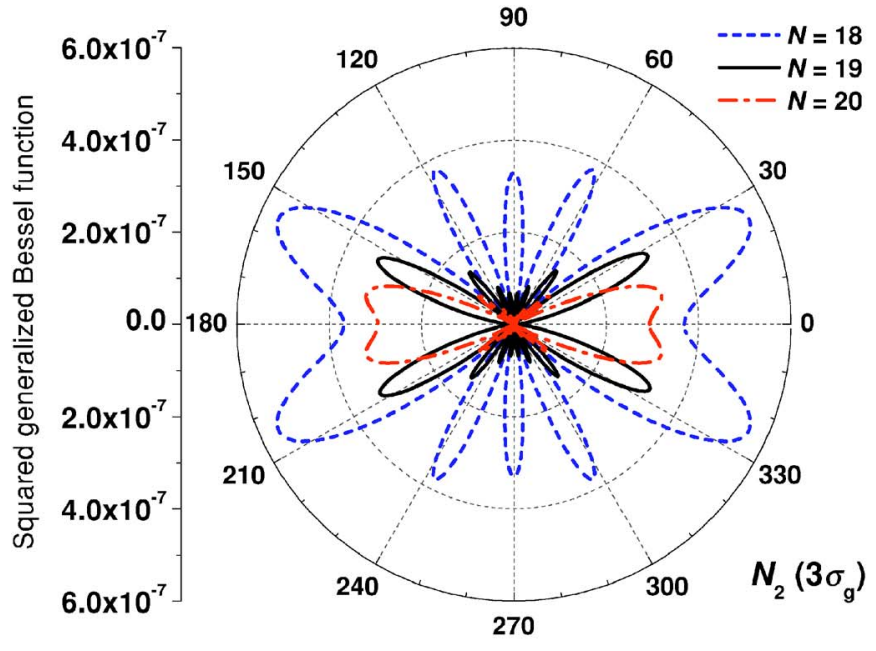

(a)

$\theta$, Angle of Photoelectron Emission (degrees)

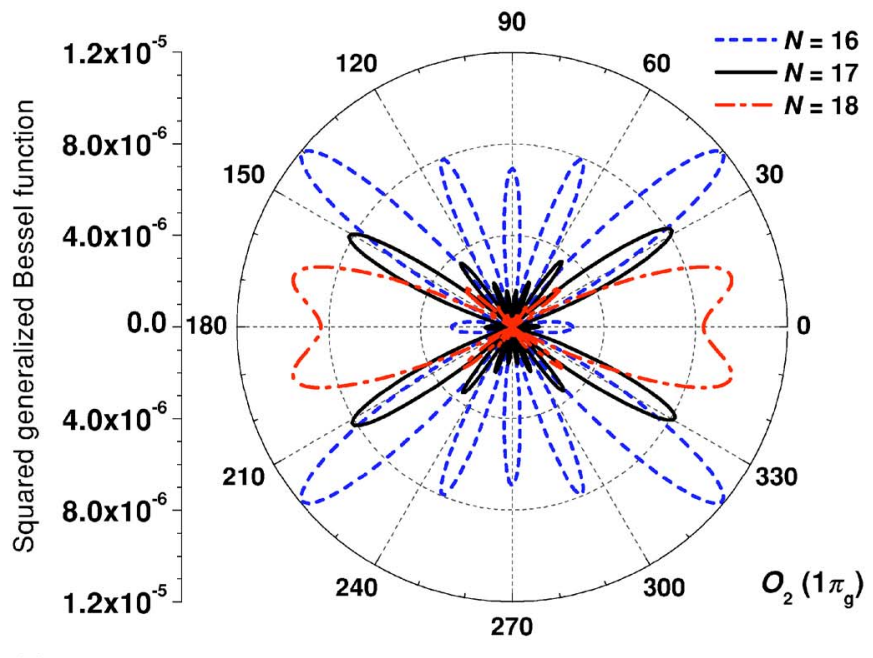

(b)

$\theta$, Angle of Photoelectron Emission (degrees)

FIG. 2. The squared generalized Bessel function $B_{-N}^{2}\left(\zeta\left(\mathbf{p}_{N}\right) ; \eta / 2\right)$ vs the angle $\theta$ of photoelectron emission with respect to polarization of Ti:sapphire laser field of fixed intensity $I$ $=2 \times 10^{14} \mathrm{~W} / \mathrm{cm}^{2}$. These angular dependencies were also calculated for three different lowest numbers $N$ of laser photons absorbed beginning from minimum one $N_{0}=\left[\left(I_{p}+U_{p}\right) / \omega\right]+1$ required for ionization of the respective outermost valence shell: (a) $3 \sigma_{g}$ in $\mathrm{N}_{2}$ molecule and (b) $1 \pi_{g}$ in $\mathrm{O}_{2}$ molecule.

is very quickly decreasing function of its order $N$ beginning from some order $N_{\max }$ [viz., $\left|B_{N}(x ; y)\right| \rightarrow 0$, for $N \gg N_{\max }$ $\sim|x|+2|y|]$. For the latter reason the number $N_{\max }$ is strongly dependent on a specified value of the parameter $\eta$ and, due to the angle-dependent argument $\zeta(\mathbf{p})=(\mathbf{E} \cdot \mathbf{p}) / \omega^{2} \sim \cos \theta$ of the generalized Bessel function (5), also on the angle $\theta$ of photoelectron emission with respect to the polarization vector $\mathbf{e}$ of incident laser field. This particularly means that, with fairly good accuracy, the number $N_{C}$ under numerical calculations of molecular ionization rates (13) and respective total PAD (12) can be approximately limited by the value $[2 \eta]$ corresponding to the number of contributing open ATI channels within the photoelectron energy region $\varepsilon_{\mathbf{p}}^{(N)} \leqslant 2 U_{p}$, where the direct ATI process under consideration is predominant. 


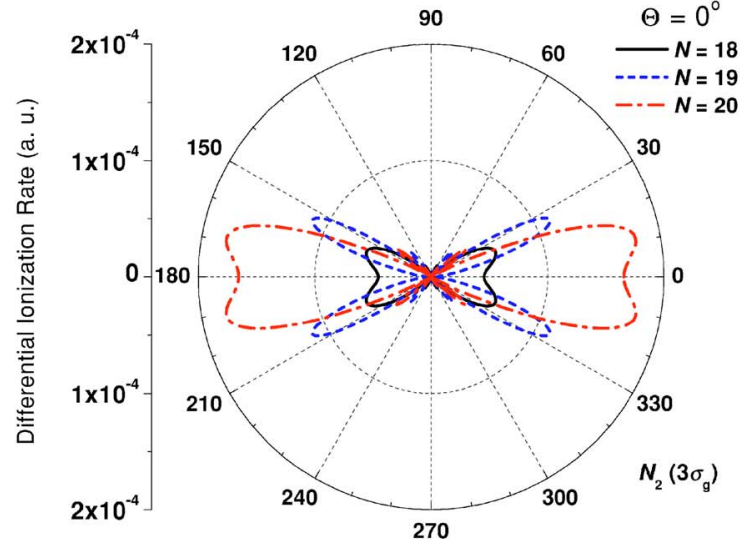

(a)

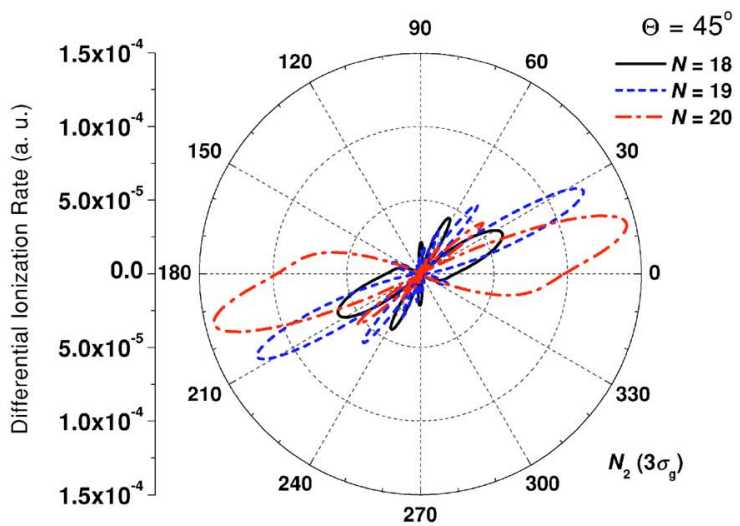

(b)

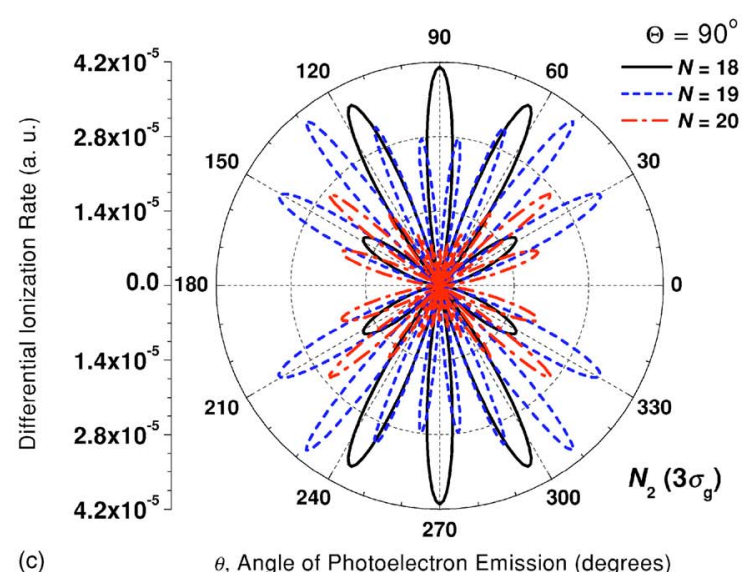

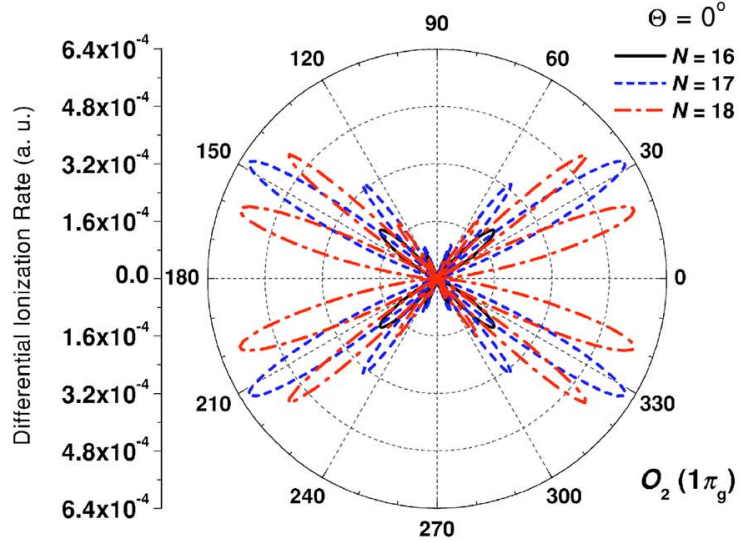

(d)

$\theta$, Angle of Photoelectron Emission (degrees)

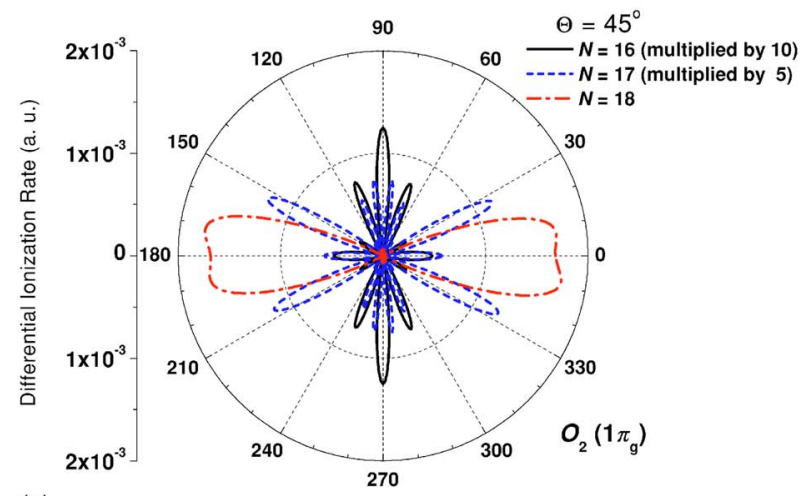

(e)

$\theta$, Angle of Photoelectron Emission (degrees)

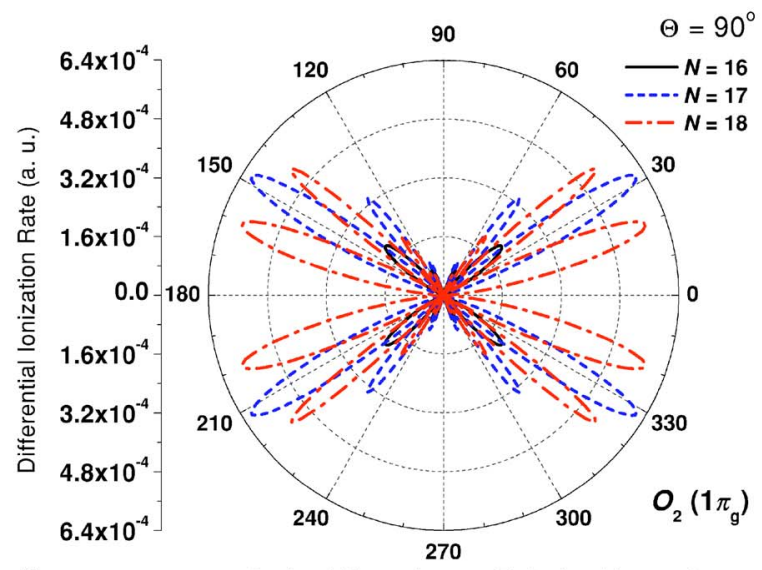

(f)

FIG. 3. $N$-photon differential molecular ionization rates (11) calculated for ionization of outermost molecular valence shells in (a-c) $\mathrm{N}_{2}$ and (d-f) $\mathrm{O}_{2}$ under the same conditions as Figs. 1 and 2 vs the angle $\theta$ of photoelectron emission with respect to incident laser field polarization, which is supposed to be lined up in horizontal direction corresponding to the angle $\theta=0$. Those angular dependencies are presented for three different orientations of the internuclear molecular axis $\Theta(0, \pi / 4$, and $\pi / 2)$ relative to polarization of the incident laser field, each for three different lowest numbers $N$ of absorbed photons required for ionization of respective outermost molecular valence shell.

According to Eqs. (20)-(23), derived for the Fourier transforms of the initial molecular state of different bonding symmetry and spatial configuration, the released photoelectron can be emitted along any arbitrary spatial direction with respect to the internuclear axis and/or incident field polarization. The latter suggests a fully three-dimensional (3D) consideration of the molecular ionization process and, unlike any ADK-based approach, allows for direct numerical calculation of molecular PADs, which seem to be of great practical interest (e.g., as a potential probe of molecular structure, intensity effects, and rotational perturbations [31]). Therefore, because the electronic distribution in $\sigma$ molecular valence shells (such as $3 \sigma_{g}$ in $\mathrm{N}_{2}$ ) is aligned (or dominated) along the internuclear axis, the angular dependence of re- 
spective Fourier transforms (20) and (21) suggests the ionization from $\sigma$ valence shells is to be predominant along the internuclear axis $\left(\theta_{\mathbf{p}} \approx 0\right)$ [see, e.g., Figs. 3(a)-3(c)]. Thus, the ionization of diatomics with the outermost $\sigma$ molecular shell (such as $\mathrm{H}_{2}$ or $\mathrm{N}_{2}$ ) is expected the largest along the internuclear axis if it is lined up with the incident field direction $\left(\Theta=0\right.$ or $\left.\theta_{\mathbf{p}}=\theta\right)$, for which, additionally, the coupling (EM interaction) of emitted photoelectron to incident laser field is also maximally strong due to the first angledependent argument $\zeta(\mathbf{p})=(\mathbf{E} \cdot \mathbf{p}) / \omega^{2} \sim \cos \theta$ of the generalized Bessel function (5). Unlike this, in $\pi$ molecular valence shells (such as $1 \pi_{g}$ in $\mathrm{O}_{2}$ ) the initial electronic cloud is aligned along a direction quite different from the internuclear axis. Particularly, the angular dependence of respective Fourier transforms (22) and (23) suggests that $1 \pi_{u}$ and $1 \pi_{g}$ valence shells are both a highly resistant to ionization along the internuclear molecular axis $\left[\sin \left(\theta_{\mathbf{p}}\right) \approx 0\right]$. Accordingly, the photoelectron emission from respective MOs [see Figs. 1(b) and $1(\mathrm{c})$ ] is always dominated along a direction different from the internuclear axis $\left[\sin \left(\theta_{\mathbf{p}}\right) \approx 1\right]$, irrespectively of its orientation relative to the incident laser field polarization. So, the form of the Fourier transform (22) prescribes the ionization from $\pi_{u}$ valence shell to be predominant along a direction perpendicular to the internuclear axis $\left(\theta_{\mathbf{p}} \approx \pi / 2\right)$. However, because of antibonding symmetry and related destructive interference along the latter direction, the ionization from the $\pi_{g}$ valence shell is to be predominant along a direction different from both the internuclear axis and any transverse direction, e.g., around the angle $\theta_{\mathbf{p}} \approx \pi / 4$ and/or $\theta_{\mathbf{p}} \approx 3 \pi / 4$ with respect to the internuclear axis (see also below). Thus, the ionization of diatomics having $\pi$ outermost valence shell is expected to be the largest if the internuclear axis is lined up along the corresponding directions with respect to incident field polarization (viz., $\Theta \approx \pi / 2$ for $\pi_{u}$ and/or $\Theta \approx \pi / 4$ for $\pi_{g}$ ). Finally, the photoelectron emission from outermost $\pi$ valence shells of homonuclear diatomics (e.g., $1 \pi_{g}$ in $\mathrm{O}_{2}$ ) lined up with the incident field direction $(\Theta=0)$ may be predominant along a spatial direction somewhat different from the above-mentioned ones (viz., $\theta=\theta_{\mathbf{p}}$ $\approx \pi / 2$ for $\pi_{u}$ and/or $\theta=\theta_{\mathbf{p}} \approx \pi / 4$ for $\pi_{g}$ ), due to a considerably weaker EM coupling to incident laser field along those directions.

Note also that, unlike $3 \sigma_{g}$ or $\pi$ molecular shells, the bonding $1 \sigma_{g}$ (e.g., in $\mathrm{H}_{2}$ ) or antibonding $2 \sigma_{u}$ valence shells can be respectively composed from two spherically symmetric $1 S$ or $2 S$ hydrogenlike AOs, which are equally well ionized, both along the internuclear axis and any transverse spatial direction. Thus, the angular behavior of amplitude of ionization from $1 \sigma_{g}$ or $2 \sigma_{u}$ is mostly determined by quite a different angular (trigonometric) factor $\cos \left[\left(\mathbf{p}_{N} \cdot \mathbf{R}_{0}\right) / 2\right]$ or $\sin \left[\left(\mathbf{p}_{N} \cdot \mathbf{R}_{0}\right) / 2\right]$, which only is angular dependent in respective Fourier transforms. Meantime, the latter trigonometric factors are also present in all Fourier transforms (20)-(23) of initially bound molecular state since they arise owing to a two-centered nature of initial molecular wave function and respective intramolecular interference of amplitudes of ionization from two separate atomic centers [25]. Particularly, the factor $\cos \left[\left(p_{N} R_{0} / 2\right) \cos \theta_{\mathbf{p}}\right]$ in (20) and (22) arises from constructive intramolecular interference that takes place un-
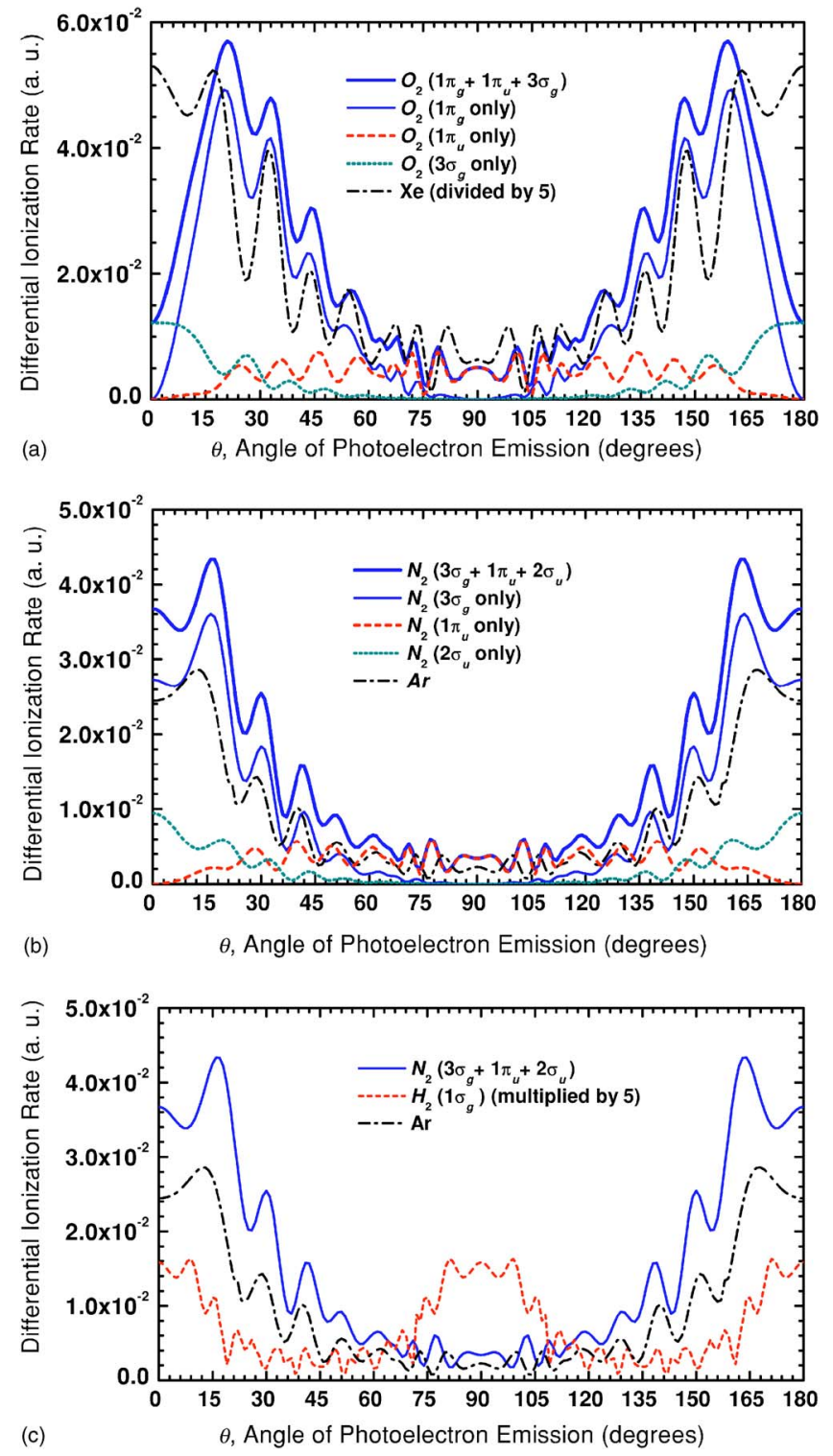

FIG. 4. The photoelectron angular distributions (PAD) (12) calculated for ionization of (a) $\mathrm{O}_{2}$, (b) $\mathrm{N}_{2}$, and (c) $\mathrm{H}_{2}$ by Ti:sapphire laser field of the same very high intensity $\left(I=1 \times 10^{15} \mathrm{~W} / \mathrm{cm}^{2}\right)$ vs the angle $\theta_{p}$ of photoelectron emission with respect to the internuclear molecular axis, which is supposed to be strongly aligned along the direction of incident laser field polarization corresponding to the angle $\theta_{p}=0$. For each of diatomics both the total molecular PAD corresponding to overall contribution from all (outermost and inner) molecular valence shells is presented (thick line) as well as the partial molecular PADs (thin lines) corresponding to separate contribution from a single respective (outermost or inner) molecular valence shells. Moreover, the atomic PADs (dashed-dotted line) produced due to ionization of respective atomic counterparts are also presented here for the convenience of direct comparison.

der ionization of molecular valence shells of bonding symmetry [22]. In other words, the mentioned intramolecular interference is to be constructive for photoelectrons emitted from bonding $\sigma_{g}$ and $\pi_{u}$ along the internuclear molecular axis [see also Figs. 3(a)-3(c)], at least, for relatively lowenergy photoelectrons (viz., $p_{N} R_{0} / 2 \leqslant 1$ ) predominantly con- 
tributing to respective total ionization rate (13). For the same reason, the ionization from $\sigma_{g}$ and $\pi_{u}$ along any transverse direction (perpendicular to the internuclear molecular axis $\left(\cos \theta_{\mathbf{p}} \approx 0\right)$ is always constructive, for both low-energy and relatively high-energy photoelectrons $\left(p_{N} R_{0} / 2 \gg 1\right)$. However, because of the presence of additional angular factor $\cos \theta_{\mathbf{p}}$ in (20), the photoelectron emission from $3 \sigma_{g}$ is suppressed along a transverse direction, whereas from $\pi_{u}$ valence shell it is strongly suppressed along the internuclear molecular axis due to the presence of $\sin \theta_{\mathbf{p}}$ in (22). Thus, we conclude that the ionization from $3 \sigma_{g}$ is predominant along the internuclear axis (although, more likely for a relatively low-energy photoelectrons), whereas the ionization from $\pi_{u}$ is predominant for all photoelectrons emitted along a transverse direction.

Conversely, the intramolecular interference of ionization from separate atomic centers is to be destructive for ionization of valence shells of antibonding symmetry, that results in arising of a quite different interference-related trigonometric factor $\sin \left[\left(p_{N} R_{0} / 2\right) \cos \theta_{\mathbf{p}}\right]$ in respective Fourier transforms, such as (21) and (23). In particular, the latter destructive interference (suppression) is to be especially prominent for all photoelectrons emitted along a transverse spatial direction, perpendicular to the internuclear axis $\left(\theta_{\mathbf{p}} \approx \pi / 2\right)$. Meanwhile, for ionization along the internuclear axis $\left(\theta_{\mathbf{p}}\right.$ $\approx 0$ ), the interference is destructive mostly for low-energy photoelectrons (viz., $\left(p_{N} R_{0} / 2 \leqslant 1\right)$ predominantly contributing to respective total ionization rate (13). Thus, since the photoelectron emission from antibonding $\pi_{g}$ is also highly suppressed along the internuclear molecular axis [due to presence of $\sin \theta_{\mathbf{p}}$ in (23)], we conclude that the ionization of $\pi_{g}$ is to be predominant along a spatial direction quite different from both the internuclear axis and/or perpendicular to it [see Figs. 3(d)-3(f)]. Unlike this, one expects that the ionization of $2 \sigma_{u}$ is most likely to occur along the internuclear molecular axis because of a highly destructive suppression of low-energy photoelectron emission along a transverse direction.

To illustrate the conclusions made above, the total PADs (12) calculated for ionization of diatomics $\mathrm{O}_{2}, \mathrm{~N}_{2}$, and $\mathrm{H}_{2}$ by the same laser pulse of high intensity and long duration are presented in Figs. 4(a)-4(c) compared to PADs produced due to ionization of respective atomic counterparts (viz., Xe for $\mathrm{O}_{2}$ and $\mathrm{Ar}$ for $\mathrm{N}_{2}$ and $\mathrm{H}_{2}$ ). Recall, that the ionization potential of $\mathrm{Xe}\left(I_{p}=12.13 \mathrm{eV}\right)$ is nearly identical to that of the outermost $1 \pi_{g}$ valence shell in $\mathrm{O}_{2} \quad\left(I_{p}^{\left(1 \pi_{g}\right)}=12.07 \mathrm{eV}\right)$, whereas the ionization potential of $\operatorname{Ar}\left(I_{p}=15.75 \mathrm{eV}\right)$ is nearly identical to that of the outermost $3 \sigma_{g}$ valence shell in $\mathrm{N}_{2}\left(I_{p}^{\left(3 \sigma_{g}\right)}=15.58 \mathrm{eV}\right)$ or $1 \sigma_{g}$ in $\mathrm{H}_{2}\left(I_{p}^{\left(1 \pi_{g}\right)}=15.43 \mathrm{eV}\right)$. Thus, all PADs presented in Figs. 4(a)-4(c) account for the overall contribution of all photoelectrons emitted from a specified molecular valence shell to final continuum states (10). Namely, these total PADs are obtained by means of summation over all contributing ATI peaks (or the so-called open ATI channels) of energies $\varepsilon_{n, \mathbf{p}}^{(N)}$, although only within the finite region $N_{0} \leqslant N \leqslant N_{0}+2 \eta$ where the direct ATI process is mostly predominant. As expected, for the particular case of strongly aligned diatomics under consideration $(\Theta=0$ and, thus $\theta=\theta_{\mathbf{p}}$ ), all the calculated PADs have azimuthal symme- try with respect to the internuclear axis. For this reason, only the dependence on the polar angle $\theta$ of photoelectron emission with respect to the incident laser field polarization is presented in Figs. 4(a)-4(c). In particular, the photoelectron emission from $\sigma$ molecular orbitals is well seen to be predominant along or around the internuclear axis. Moreover, since the internuclear axis is supposed to be coincident with the polarization of incident laser field, the photoelectron emission from $\sigma$ valence shell is highly suppressed along the direction perpendicular to the internuclear axis. Unlike this, the photoelectron emission from $\pi$ valence shells is well seen to be highly suppressed along the internuclear molecular axis, irrespective of the direction of incident laser field polarization. The calculated PADs presented in Figs. 4(a) and 4(b) and corresponding to ionization of $\pi_{u}$ valence shells do demonstrate such a high suppression in ionization along the internuclear molecular axis as well as no suppression in photoelectron emission along the transverse direction due to a bonding symmetry and related constructive interference of ionization contributions from separate atomic centers. Unlike $\pi_{u}$, the emission of photoelectrons from $\pi_{g}$ valence shell is also strongly suppressed along the direction perpendicular to the incident laser field polarization because of antibonding symmetry and related destructive interference of ionization contributions from separate atomic centers. As a result, the photoelectrons are predominantly emitted from $1 \pi_{g}$ valence shells along the direction around the angles $\theta_{\mathbf{p}} \approx 25^{\circ}$ and $\theta_{\mathbf{p}} \approx 155^{\circ}$ (or, more exactly, within the angle regions $15^{\circ}$ $\leqslant \theta_{\mathbf{p}} \leqslant 45^{\circ}$ and $135^{\circ} \leqslant \theta_{\mathbf{p}} \leqslant 165^{\circ}$ ).

Thus, the strong-field amplitudes of molecular ionization derived within the currently proposed SFA-LCAO approach generally depend on a variety of the problem parameters (e.g., laser frequency and intensity) and structure features of the molecular system under ionization (such as structure of molecular valence shells and their ionization potential, bonding symmetry and spatial configuration with respect to the internuclear molecular axis and orientation with respect to the incident laser field polarization, etc.). Accordingly, the phenomenon of suppressed or enhanced molecular ionization compared to ionization of a counterpart atomic system is not to be generally a result of only one of these important features alone, but rather the result of interplay of all these factors put together, so that each of them requires special and careful consideration. According to the SFA-LCAO approach, all the model-related derived analytical expressions (11)-(13) and (20)-(23) do suggest fully 3D treatment of the molecular ionization process that implies the photoelectron emission along any (arbtrary) spatial direction relative to the incident laser field polarization which can be in general randomly oriented with respect to the internuclear molecular axis. Nonetheless, let us suppose further the internuclear axis to be strongly aligned along the polarization of incident laser field (i.e., $\Theta=0$ and $\theta=\theta_{\mathbf{p}}$ ) as the orientation effects in strong-field molecular ionization are deserving of special detailed consideration, elsewhere in our further publications. The latter oversimplifying supposition is made just to reduce the number of all these influencing factors to fewer ones, moreover, it is also based on the underlying physical mechanism conventionally referred to as a dynamic alignment in current literature [32]. In the meantime, the approximation 
made seems to be sufficiently well justified (see, e.g., [33]) for laser pulses of sufficiently long duration and also supported by other alternative calculations (e.g., within twodimensional (2D) extension of ADK consideration [34]) in which the orientation averaging effects proved to be negligible and, thus, quite insufficient to provide an observed high suppression in ionization of $\mathrm{O}_{2}$ versus its atomic counterpart Xe. As was firmly established earlier [35], the field associated with intense, linearly polarized laser radiation of picosecond pulse duration induces sufficiently strong torques on an initially randomly oriented ensemble of linear diatomic molecules, so that reorientation of internuclear axis occurs. In later experiments [36] with a number of heavy diatomic molecules irradiated by sub- 100 fs pulse of a Ti:sapphire laser, it was also shown that studying ionization without alignment does require only a molecule with large moment of inertia and small anisotropic polarizability [37], so that the experiment [38] shows clear signs of dynamic alignment of lighter molecules (such as $\mathrm{H}_{2}, \mathrm{~N}_{2}$, and $\mathrm{O}_{2}$ ), which are forced into alignment by the sub-100 fs laser pulse.

When studying the strong-field ionization process, the major difficulty with molecules is to deal with both the electronic and nuclear degrees of freedom. Therefore, the latter is often neglected, through the use of the Born-Oppenheimer approximation, keeping the nuclei fixed and thus ignoring the role of other possible contributing channels-the dissociative ionization and Coulomb explosion. Historically, the three channels-ionization, dissociation, and Coulomb explosion-were normally treated as sequential processes [39]. The latest developments, however, suggest that this is not always true. According to an analysis [40] based on a one-dimensional (1D) model of the response of a simple molecular ion to an intense infrared laser pulse, it is necessary to include the motion of the nuclei in order to properly address the question of the competition between the different processes: excitation, ionization (leading to Coulomb explosion), and dissociation. The latter, in particular, depends critically on the duration of the laser pulse and also on the absolute phase of the laser. Depending on the exact pulse parameters, the ionization, dissociative ionization, and even a tiny amount of Coulomb explosion occur in parallel. In the case of incident laser pulse parameters (intensity $I \leqslant 1$ $\times 10^{15} \mathrm{~W} / \mathrm{cm}^{2}$ and pulse duration $\tau \leqslant 100 \mathrm{fs}$ ) and sequential processes under consideration (when, for example, the ionization step followed by dissociation or by a second ionization step), the ionization process generally saturates at a lower intensity than the second step. As long as the process of molecular ionization under study approaches saturation, it is quite common that the signals from the consecutive steps are still far from saturation and differ by orders of magnitude, so that the respective contribution from the latter consecutive processes can be ignored. Thus, despite the fact that dissociation certainly occurs too, it becomes significant and saturates at a much higher laser intensity $\left(I \geqslant 10^{15} \mathrm{~W} / \mathrm{cm}^{2}\right)$, which is beyond the scope of this paper.

Nonetheless, including the nuclei motion in proper consideration is generally feasible within the framework of the currently applied SFA-LCAO approach, but this additionally requires preliminary knowledge of available reliable data (previously calculated or measured in experiment) related to

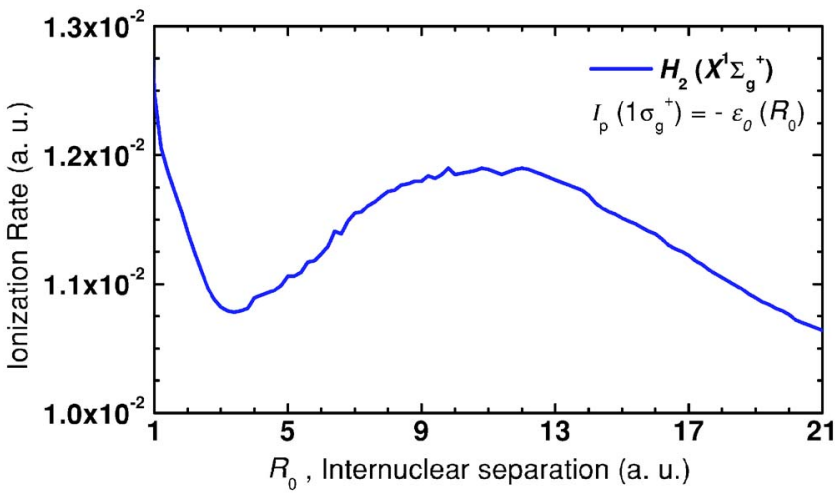

FIG. 5. The calculated molecular ionization rate (13) for the laser-irradiated $\mathrm{H}_{2}$ molecule, under the same conditions as in Fig. 4 vs the internuclear separation $R_{0}$ beginning from equilibrium one $R_{e}=0.742$ a.u. The presented results were calculated taking into account the $R_{0}$ dependence of the molecular binding energy $\varepsilon_{0}\left(R_{0}\right)$ corresponding to the ground $X^{1} \Sigma_{g}^{+}$state.

dependence of molecular binding energy on internuclear separation $R_{0}$. To the best of our knowledge, the latter dependence is reliably known only for the simplest diatomics $\left(\mathrm{H}_{2}^{+}\right.$or $\left.\mathrm{H}_{2}\right)$, and Fig. 5 presents our numerical results for ionization of the ground $X^{1} \Sigma_{g}^{+}$state of $\mathrm{H}_{2}$ and respective $R_{0}$ dependence of the calculated ionization rate (13). These results are generally consistent with similar $R_{0}$-dependent behavior of the ionization rate revealed in [40] under 1D numerical simulation of strong-field ionization of diatomic ion $\mathrm{H}_{2}^{+}$. Particularly, Fig. 5 demonstrates a noticeable (although, not so very impressive, as reported in [40]) transient enhancement of molecular ionization rate for expanding molecules. As in [40], after reaching a local maximum at $R_{0}$ $\approx 9$ a.u., the ionization rate also gradually decreases under further $R_{0}$ increasing. Note also that unlike [40], there is some initial (though, also a transient) decrease of ionization rate clearly visible within the domain $R_{e} \leqslant R_{0} \leqslant 3$ a.u. in Fig. 5. The latter transient decrease and the following transient enhancement of the $\mathrm{H}_{2}$ ionization rate mentioned above is, however, in qualitative agreement with similar behavior of electron-nuclear dynamics of the multiphoton $\mathrm{H}_{2}^{+}$dissociative ionization numerically calculated in [41] [see, e.g., Fig. 5(b) presented therein] and allows for transparent interpretation within the currently proposed model. Namely, the initial (transient) and final decrease of ionization rate in expanding $\mathrm{H}_{2}$ is explained by respective reduction of contribution from low-energy photoelectrons (viz., of momentum $p_{N} \leqslant 2 / R_{0}$ ), only for which, because of the trigonometric factor $\cos \left[\left(p_{N} R_{0} / 2\right) \cos \theta_{\mathbf{p}}\right]$, the intramolecular interference in bonding $1 \sigma_{g}$ along the molecular axis $\left(\cos \theta_{\mathbf{p}} \approx 1\right)$ is still constructive. Meanwhile, in expanding diatomic $\mathrm{H}_{2}$ the respective $R_{0}$-dependent molecular ionization potential $I_{p}\left(R_{0}\right)$ gradually decreases, thereby reducing the respective number $N_{0}^{\left(1 \sigma_{g}\right)}$ of absorbed photons minimally required for ionization of $1 \sigma_{g}$. This leads to a considerably enhanced ionization rate, insomuch as under further expansion of $\mathrm{H}_{2}$ the latter enhancement may become temporarily prevailing over the opposite mentioned effect arising from reduced contribution of low-energy photoelectrons because of destructive closing of 

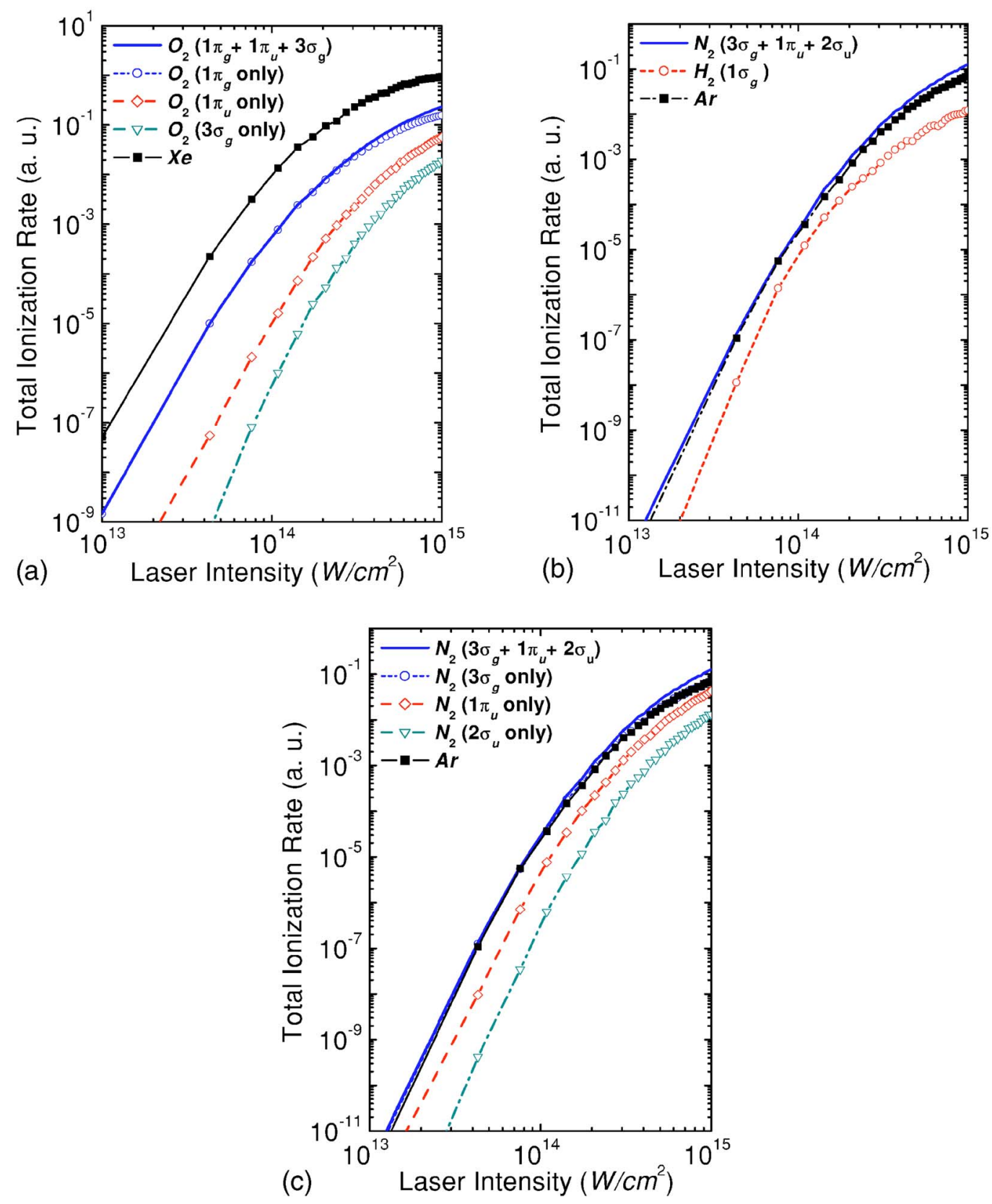

FIG. 6. The molecular ionization rates (13) calculated for laser-irradiated diatomics (a) $\mathrm{O}_{2}$, (b) $\mathrm{H}_{2}$, and (c) $\mathrm{N}_{2}$ vs the intensity $I$ of Ti:sapphire laser radiation, assuming that the internuclear molecular axis is strongly aligned along the laser field polarization. For each of the diatomics, both the total ionization rate (thick solid line) corresponding to overall contribution from outermost and the inner molecular valence shells (i.e., $1 \pi_{g}, 1 \pi_{u}$, and $3 \sigma_{g}$ in $\mathrm{O}_{2}$ or $3 \sigma_{g}, 1 \pi_{u}$, and $2 \sigma_{u}$ in $\mathrm{N}_{2}$ ) is presented as well as the respective partial ionization rates (thin lines) corresponding to separate contribution from a single (outermost or inner) molecular valence shell under consideration. Moreover, the calculated atomic ionization rates produced due to ionization of the respective atomic counterpart are also presented (filled squares). All these presented results are to be compared to the respective results of numerous earlier alternative calculations (e.g., Fig. 3 presented in the first paper of Ref. [22]) and reported in relevant experiments (e.g., presented in Figs. 1 and 2 of Ref. [14] and Fig. 1 in Ref. [13].

some low-energy ATI channels. The latter explains a subsequent transient rising of the ionization rate within an intermediate region ( 3 a.u. $\leqslant R_{0} \leqslant 9$ a.u.), although, under further expansion $\left(R_{0} \geqslant 9\right.$ a.u.), the mentioned enhancement is getting down because of a negligible variation of molecular ionization potential.

Since for other diatomics under consideration the value of molecular binding energy is reliably known only at equilib- rium internuclear separation $R_{e}$, our current consideration is restricted to the fixed-nuclei approximation, so that in this paper we will further focus on the electron ionization aspect only at $R_{0}=R_{e}$. The SFA-LCAO model related numerical results for molecular ionization rates (13) of strongly aligned diatomics $\left(\mathrm{O}_{2}, \mathrm{~N}_{2}\right.$, and $\left.\mathrm{H}_{2}\right)$, calculated according to derived analytical expressions (20)-(26), are presented versus the intensity of an incident Ti:sapphire laser field (of wavelength 
$\lambda=800 \mathrm{~nm}$ ) in the Figs. 6(a)-6(c) as well as the ionization rates of respective atomic counterparts. These results show clear signs of a high suppression in ionization of $\mathrm{H}_{2}$ and $\mathrm{O}_{2}$ (with respect to ionization in $\mathrm{Ar}$ and $\mathrm{Xe}$, respectively) as well as no suppression or (beginning from $5 \times 10^{14} \mathrm{~W} / \mathrm{cm}^{2}$ ) even an enhanced ionization of $\mathrm{N}_{2}$ molecule relative to $\mathrm{Ar}$, that all is in a qualitative accordance with relevant experimental data observed in experiments [13-16]. Particularly, the presently calculated suppression in total ionization rates of $\mathrm{O}_{2}$ with respect to $\mathrm{Xe}$ has been found to be well over about one order of magnitude in the lower-intensity region $\left(10^{13} \mathrm{~W} / \mathrm{cm}^{2} \leqslant I\right.$ $\leqslant 2 \times 10^{14} \mathrm{~W} / \mathrm{cm}^{2}$ ) and becomes a bit smaller in the higherintensity region $\left(I \geqslant 5 \times 10^{14} \mathrm{~W} / \mathrm{cm}^{2}\right)$ due to increased contributions from inner $1 \pi_{u}\left(I_{p}^{\left(1 \pi_{u}\right)}=16.26 \mathrm{eV}\right)$ and $3 \sigma_{g}\left(I_{p}^{\left(3 \sigma_{g}\right)}\right.$ $=18.18 \mathrm{eV}$ ) valence shells equally well taken into account and also separately represented in Fig. 6(a). Thus, the latter contributions of two other closest inner valence shells to the total calculated ionization rate of $\mathrm{O}_{2}$ proved to always be negligibly small compared to predominantly the contributing outermost $1 \pi_{g}$ valence shell. Also, Fig. 6(b) shows a noticeable suppression in ionization of $\mathrm{H}_{2}$ with respect to its diatomic counterpart $\mathrm{N}_{2}$ and atomic counterpart $\mathrm{Ar}$, so that the latter calculated suppression relative to $\mathrm{Ar}$ is about of the same value (less than about one order of magnitude) within the low-intensity region $10^{13} \mathrm{~W} / \mathrm{cm}^{2} \leqslant I \leqslant 2 \times 10^{14} \mathrm{~W} / \mathrm{cm}^{2}$. Thus, being a noticeably smaller than suppression in ionization of $\mathrm{O}_{2}$ relative to Xe [suggested in Fig. 1(a)], the latter suppression in ionization of $\mathrm{H}_{2}$ is getting considerably more pronounced within the relatively high-intensity region $I \geqslant 2$ $\times 10^{14} \mathrm{~W} / \mathrm{cm}^{2}$ under consideration. Such a behavior is also fairly well consistent with respective dependence of the molecular ionization rate on incident laser intensity revealed in recent experiments for ionization of $\mathrm{D}_{2}$ (see, e.g., Fig. 5 in [16]), although in a contradiction to relevant results calculated within the MO-ADK model of molecular strong-field ionization [24] (at least, within the strong-field domain only where the validity of the ADK theory is well justified, see, e.g., Fig. 6 presented therein).

In the meantime, the calculated total ionization rates for $\mathrm{N}_{2}$ as well as the respective separate contributions of the outermost $3 \sigma_{g}$ and two inner $1 \pi_{u}\left(I_{p}^{\left(1 \pi_{u}\right)}=16.96 \mathrm{eV}\right)$ and $2 \sigma_{u}$ $\left(I_{p}^{\left(2 \sigma_{u}\right)}=18.73 \mathrm{eV}\right)$ valence shells show quite a different relative behavior compared to companion Ar atom. So, Fig. 6(c) also demonstrates that within a broad region of laser intensity values $\left(I \leqslant 5 \times 10^{14} \mathrm{~W} / \mathrm{cm}^{2}\right)$ the relative contribution from inner shells to ionization of $\mathrm{N}_{2}$ is also considerably less than that of the outermost $3 \sigma_{g}$, although slightly larger as compared to that from $1 \pi_{u}$ and $3 \sigma_{g}$ inner shells in ionization of $\mathrm{O}_{2}$. According to the results presented in Fig. 6(c), owing to the contribution from inner shells, the ionization of $\mathrm{N}_{2}$ is just a slightly enhanced relative to Ar, especially within the region of relatively low and moderate laser intensity. However, in a high-intensity region (over than about 5 $\times 10^{14} \mathrm{~W} / \mathrm{cm}^{2}$ ) the enhanced ionization of $\mathrm{N}_{2}$ relative to $\mathrm{Ar}$ becomes more prominent because of the fast increase of relative contributions from the $1 \pi_{u}$ and $2 \sigma_{u}$ inner shells when the laser intensity increases. Thus, the currently applied SFALCAO model also suggests the background mechanism underlying (at least, within the high-intensity region) an en- hanced ionization in $\mathrm{N}_{2}$, which is thus due to ionization of two other (inner) contributing valence shells. Otherwise, without taking any contribution of inner molecular shells involved into ionization, the contribution only from ionization of the $3 \sigma_{g}$ shell would just result in no suppression in ionization of $\mathrm{N}_{2}$ relative to Ar.

Like the MO-SFA-based molecular ionization model [22], the currently applied SFA-LCAO model also suggests the similar predominant background mechanism underlying the high suppression in ionization of $\mathrm{O}_{2}$ and/or no suppression present in ionization of $\mathrm{N}_{2}$. Namely, the reason of high suppression in ionization of $\mathrm{O}_{2}$ is also identified here as a highly destructive interference of ionization from separate atomic centers due to antibonding symmetry of a predominantly contributing outermost valence shell. Because of the presence of the trigonometric factor $\sin \left[\left(\mathbf{p}_{N} \cdot \mathbf{R}_{0}\right) / 2\right]$ in the Fourier transform (23) of the initial two-centered molecular state corresponding to the predominantly contributing antibonding $1 \pi_{g}$ outermost valence shell of $\mathrm{O}_{2}$, the respective total ionization rate contains the interference-related factor

$$
2\left|\sin \left[\left(\mathbf{p}_{N} \cdot \mathbf{R}_{0}\right) / 2\right]\right|^{2}=1-\cos \left[\left(\mathbf{p}_{N} \cdot \mathbf{R}_{0}\right)\right] .
$$

Thus, the latter interference gives rise to a suppression of few ATI photoelectron peaks $\left(p_{N} R_{0} \cos \theta_{\mathbf{p}} \leqslant 1\right)$ of the lowest energy corresponding to a few lowest values of number $N$ of incident photons absorbed over the minimal one $N_{0}^{(n)}=\left[\left(I_{p}^{(n)}\right.\right.$ $\left.\left.+U_{p}\right) / \omega\right]+1$ required for ionization. The mentioned high suppression is well illustrated by Fig. 7(a), where the molecular photoelectron spectra corresponding to ionization of $\mathrm{O}_{2}$ are represented in the form of integral ionization rates $R_{N}^{(n)}\left(p_{N}, \eta\right)$ [Eq. (11)] calculated both for all and separately for each of the contributing valence shells. Namely, the lowest-energy ATI peaks with $N=N_{0}^{\left(1 \pi_{g}\right)}=43$ and 44 presented in Fig. 6(a) are well seen to be noticeably suppressed in the photoelectron spectrum corresponding to the ionization of the antibonding $1 \pi_{g}$ valence shell. Figure 7 (a) also demonstrate that the respective low-energy ATI peaks are not considerably suppressed in integral photoelectron spectra corresponding to ionization of spherically symmetric initially bound atomic state of $\mathrm{Xe}$ and, due to a considerably higher height (intensity), those low-energy ATI peaks predominantly contribute to the total atomic ionization rate. The latter feature in atomic photoelectron spectra is mainly related to the asymptotic behavior of generalized Bessel function $B_{-N}(\zeta(\mathbf{p}) ; \eta)$ for very large order $N$ and small first argument $\zeta\left(\mathbf{p}_{N}\right)=\left(\mathbf{E} \cdot \mathbf{p}_{N}\right) / \omega^{2} \sim\left(E / \omega^{2}\right) \sqrt{2\left(N \omega-I_{p}-U_{p}\right)}$, which is increasing with an increase of the order $N$ corresponding to the total number of incident photons absorbed. Thus, despite $\zeta\left(\mathbf{p}_{N}\right) \leqslant 1$ for a few lowest-energy photoelectron peaks of order beginning from a minimum one $N_{0}$ (the minimum number of absorbed photons required for ionization), this does not necessarily mean that $B_{-N}\left(\zeta\left(\mathbf{p}_{N}\right) ; \eta\right)$ is to be initially a decreasing function of the order $N \geqslant N_{0} \gg 1$. In the meantime, the respective few lowest-energy ATI peaks are noticeably suppressed in photoelectron spectra of $\mathrm{O}_{2}$ due to the antisymmetric nature of the outermost (and predominantly contributing) antibonding $1 \pi_{g}$ shell and related destructive 

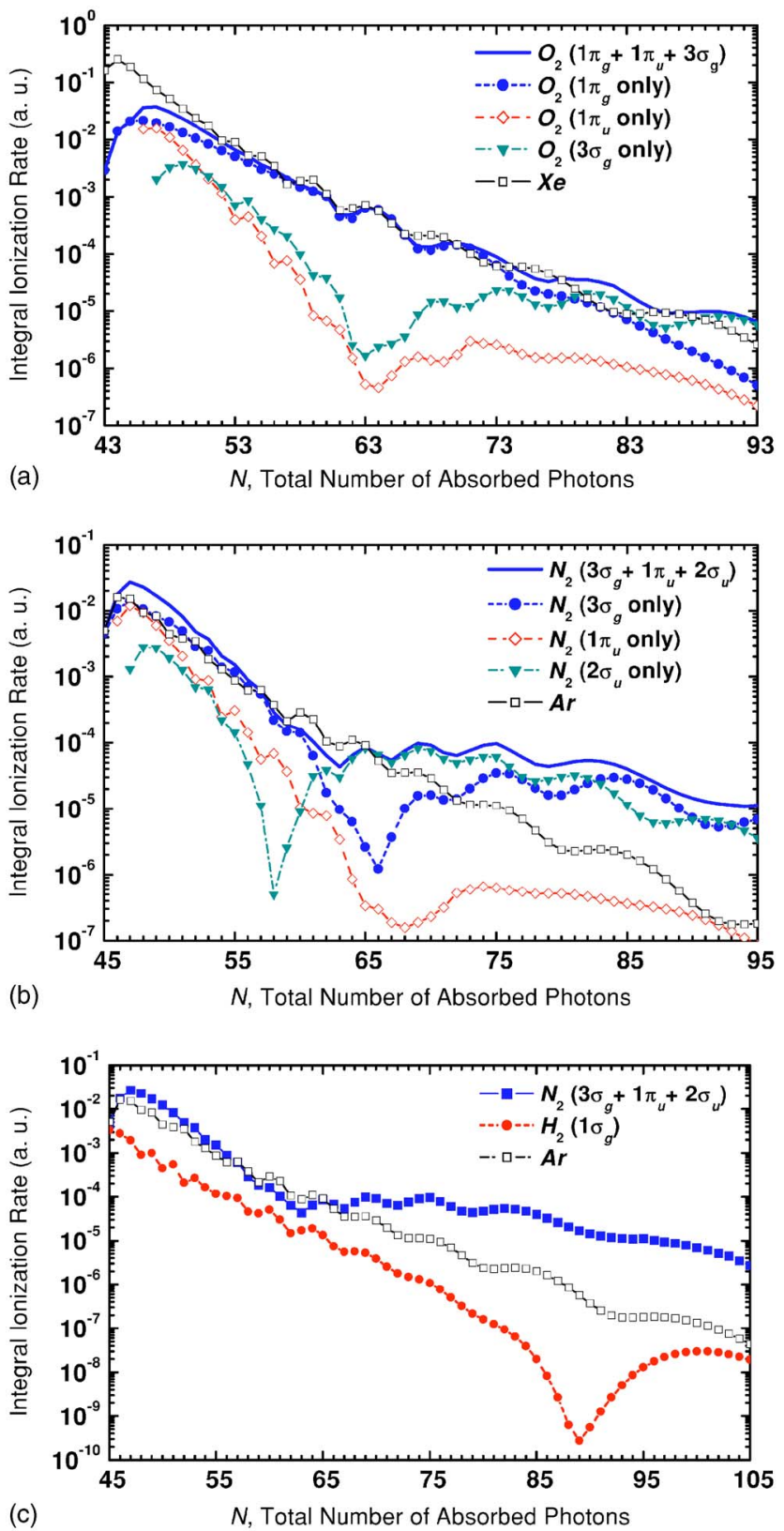

FIG. 7. The molecular integral photoelectron spectra of laserirradiated diatomics (a) $\mathrm{O}_{2}$, (b) $\mathrm{N}_{2}$, and (c) $\mathrm{H}_{2}$ calculated under the same conditions as Fig. 4 and represented as integral $N$-photon ionization rates $R_{N}^{(n)}\left(p_{N}, \eta\right)$ [Eq. (11)] vs the total number $N$ of incident laser photons absorbed. For each of diatomics both the total photoelectron spectrum corresponding to the overall contribution from outermost and inner molecular valence shells is presented (thick solid line) as well as the partial photoelectron spectra (thin lines) corresponding to the separate contribution from a single (outermost or inner) molecular valence shell under consideration. Moreover, the calculated atomic integral photoelectron spectra due to ionization of the respective atomic counterpart are also presented here (open squares) for the convenience of direct comparison.

interference. In other words, the interference-related suppression of those low-energy ATI peaks and their considerably reduced contribution to the total ionization rate is the reason for which the ionization of $\mathrm{O}_{2}$ is highly suppressed.
Figures $7(a)-7(\mathrm{c})$ also demonstrate clearly the main reason for which the relative contributions from inner molecular valence shells to the total ionization rate are generally smaller. Namely, because of a larger value of the ionization potential, a number of several lowest-energy ATI channels are closed for ionization of inner molecular shells, whereas these predominantly contributing channels are still open for the outermost shell and responsible for the presence of a few lowest-energy ATI peaks in the respective photoelectron spectra. The closing of those lowest-energy ATI channels under ionization of respective inner molecular shell and related lack of partial contributions from corresponding photoelectron peaks (though, predominantly contributing to the ionization rate of the outermost molecular shell) is, thus, identified as the background mechanism underlying a relatively small contribution to the ionization rate from inner molecular shells. Particularly, there are several lowest ATI peaks in $\mathrm{O}_{2}$ photoelectron spectra corresponding to $N=43,44$, and 45 lacking in spectra due to separate ionization of inner $1 \pi_{u}$ (viz., $N=43$ and 44 ) and $3 \sigma_{g}$ (viz., $N=43,44$, and 45 ) molecular shells calculated under the same laser pulse parameters. There are also few lowest ATI peaks in $\mathrm{N}_{2}$ photoelectron spectra corresponding to $N=45$ and 46 lacking in ATI spectra due to separate ionization of inner $1 \pi_{u}$ and $2 \sigma_{u}$ shells.

Another remarkable feature well recognized in molecular photoelectron spectra corresponding to ionization of molecular valence shell of bonding symmetry, such as $3 \sigma_{g}$ and $1 \pi_{u}$ in $\mathrm{N}_{2}$ and $\mathrm{O}_{2}$ or $1 \sigma_{g}$ in $\mathrm{H}_{2}$, and presented in Figs. 7(a)-7(c) is a pronounced deep minimum in the intermediate (highenergy) domain, where the direct ATI process is, however, still predominant. Even for the same laser pulse parameters, the position of the minimum is different for different valence shell and proved to be also strongly dependent on respective binding energy, the internuclear separation $R_{0}$ and the angle $\Theta$ of the internuclear axis orientation with respect to polarization of incident laser field (i.e., $\left.\cos \Theta=\mathbf{e} \cdot \mathbf{R}_{0}\right) / R_{0}$ ). Being expressed in total number $N$ of incident photons absorbed, the position of the minimum in all calculated spectra has been ascertained to obey the following relation:

$$
N_{\min }^{(n)}=N_{0}^{(n)}+\pi^{2} /\left(2 \omega R_{0}^{2} \cos ^{2} \Theta\right)
$$

where $N_{0}^{(n)}$ is the introduced earlier minimum number of photons required to overcome the field-induced ionization threshold corresponding to $n$th valence shell. The origin of the minimum is strongly related to the two-centered nature of the initial bound molecular state under ionization and can be explained in terms of the respective intramolecular interference of ionization from two atomic centers separated by the internuclear distance $R_{0}$. Because of the presence of the cosine interference-related trigonometric factor in the Fourier transforms (20) and (22) or the factor $\cos ^{2}\left[\left(\mathbf{p}_{N} \cdot \mathbf{R}_{0}\right) / 2\right]$ in the respective molecular ionization rate corresponding to bonding valence shell, the ionization from separate AO can also be highly destructive for large photoelectron momenta satisfying the condition 


$$
\begin{aligned}
2 \cos ^{2}\left[\left(\mathbf{p}_{N} \cdot \mathbf{R}_{0}\right) / 2\right] & =1+\cos \left(\mathbf{p}_{N} \cdot \mathbf{R}_{0}\right) \equiv 1+\cos \left(p_{N} R_{0} \cos \theta_{\mathbf{p}}\right) \\
& \approx 0 .
\end{aligned}
$$

The latter condition can be rewritten in the different equivalent form

$$
\begin{aligned}
p_{N} R_{0} \cos \theta_{\mathbf{p}}= & p_{N} R_{0}(\cos \theta \cos \Theta+\sin \theta \sin \Theta \cos \varphi) \\
& \approx \pi(2 l+1), \quad l=0,1,2 \cdots
\end{aligned}
$$

or, after averaging over all angles $(\theta$ and $\varphi)$ of photoelectron emission with respect to incident field polarization, one can derive for the position of the first minimum (viz., $l=0$ ) in integral molecular photoelectron spectra (i.e., integrated over all angles of photoelectron emission)

$$
\frac{p_{N_{\min }}^{2}}{2}=\left(N_{\min }-N_{0}\right) \omega=\frac{\pi^{2}}{2 R_{0}^{2} \cos ^{2} \Theta} .
$$

For the particular case of ionization of strongly aligned diatomics under consideration (viz., $\cos \Theta \approx 0$ ), one can evaluate and verify that the position of the first minimum in all photoelectron spectra corresponding to ionization of each molecular bonding valence shell presented in Figs. 6(a)-6(c) is in excellent accordance with what is prescribed by Eq. (28). The case of $2 \sigma_{u}$ valence shell in $N_{2}$ is exceptional since the similar pronounced minimum in the respective molecular photoelectron spectrum is rather related to the particular analytical form (21) of the Fourier transform $\Phi_{2 s}\left(\mathbf{p}_{N}\right) \sim\left(\kappa_{n}^{2}\right.$ $\left.-p_{N}^{2}\right)$ for $2 s$ AO from which the molecular $2 \sigma_{u}$ valence shell is composed. Thus, the latter minimum has nothing to do with the destructive interference for large photoelectron momenta under discussion, so that its position can be adequately found by means of quite a different relation $N_{\min }^{\left(2 \sigma_{n}\right)}$ $=N_{0}^{\left(2 \sigma_{u}\right)}+\kappa_{n}^{2} /(2 \omega)$. To conclude, all the calculated molecular photoelectron spectra presented in Figs. 6(a)-6(c) show the general form to be in fairly good accordance with similar ones calculated in [23] and also demonstrate a behavior quite consistent with spectra observed in molecular ATI experiments (e.g., [42]).

\section{CONCLUSION}

The strong-field ionization in a number of light homonuclear diatomic molecules irradiated by an intense laser radiation field was considered theoretically and studied numerically within the framework of the proposed SFA-LCAO model based on a conventional strong-field approximation supplemented by the linear combination of atomic orbitals (LCAO) and molecular orbitals (MO) method invoked for analytical reproducing the one-electron wave function of an initially bound molecular state under ionization. The latter wave function is approximately reproduced as two-centered MO consisting of either symmetric (for bonding state) or antisymmetric (for antibonding state) superposition of two predominantly contributing hydrogenlike AOs shifted to each other by equilibrium internuclear separation $R_{e}$. The form of these two AOs is generally different for molecular shells (such as $\sigma_{g}, \pi_{u}$ and $\pi_{g}$ ) of various spatial (geometrical) configuration and chosen to fairly well reproduce the distribution of the respective electronic density. Besides the proper bonding symmetry of the molecular shell under ionization, the proposed SFA-LCAO model also provides one with an adequate (though, also a priori approximate) fully 3D description of both an initially bound molecular state and the entire strong-field molecular ionization process. The latter process is described as a highly nonperturbative and pure SAE response to incident laser field corresponding to a superposition of amplitudes of ionization from two separate atomic centers. Unlike the similar model [22] developed earlier, the spatial configuration of molecular orbitals with respect to the internuclear axis is taken into completely SAEbased analytical consideration and, wherever appropriate, the additional contribution of other (inner) valence shells of larger binding energy is incorporated as well. Moreover, unlike any ADK-based theory, the applied approach suggests a fully $3 \mathrm{D}$ consideration, so that all the model-related analytical expressions are derived in a general form assuming an arbitrary direction of photoelectron emission with respect to incident field polarization or the internuclear axis. Accordingly, the derived expressions allow for representation of differential and integral molecular ionization rates (as well as for respective PADs) in a closed and compact analytical form available for direct numerical calculations and transparent interpretation (including the so-called orientational effects).

For the particular case of strong molecular alignment, which was only considered under the final numerical calculations, the proposed model also predicts a high suppression in ionization of $\mathrm{O}_{2}$ and $\mathrm{H}_{2}$ (as well as no suppression for $\mathrm{N}_{2}$ ) compared to their "companion" atoms and proposes the background mechanism underlying this phenomenon. The latter is currently identified as the result of an interplay of two main (and equally important) reasons. Namely, according to the SFA-LCAO model, the origin of enhanced (or suppressed) ionization in homonuclear diatomics is attributed to (i) constructive (or destructive) intramolecular interference in the outermost $\mathrm{MO}$ under ionization (such as in the MO-SFA model [22]); (ii) particular spatial (geometrical) configurations of the outermost $\mathrm{MO}$ and its orientation relative to the internuclear axis and laser field polarization (such as in MO-ADK model [24]). So, a high suppression in ionization of $\mathrm{O}_{2}$ compared to companion $\mathrm{Xe}$ is explained mostly by a particular configuration of the outermost $1 \pi_{g} \mathrm{MO}$, whose $2 p_{x, y}$ AOs are oriented normally to the molecular axis and, thus, contribute to ionization negligibly due to the EM coupling to incident laser field is the most weak along a transverse direction $\left(\cos \theta_{\mathbf{p}} \approx 0\right)$ under the case of molecular alignment. In addition, because of the presence of the sine trigonometric factor in (23), the intramolecular interference is destructive for all photoelectrons emitted from $1 \pi_{g} \mathrm{MO}$ along a transverse direction, whereas, for ionization along the internuclear axis, the intramolecular interference is destructive only for low-energy photoelectrons, the suppression of which [normally contributing predominantly to respective molecular ionization rate (13)] leads to a high suppression in ionization of $\mathrm{O}_{2}$. In the meantime, the suppression in ionization of $\mathrm{H}_{2}$ (compared to Ar) is currently identified as the mostly geometrical phenomenon, i.e., related to spatial configuration of its $1 \sigma_{g} \mathrm{MO}$ consisting of spherically symmetric $1 S \mathrm{AOs}$, from which the photoelectron emission is equally 
possible in any spatial direction. Moreover, because of the cosine trigonometric factor $\cos \left(p_{N} \cdot R_{0} \cdot \cos \theta_{\mathbf{p}}\right)$, the related intramolecular interference in ionization of bonding $1 \sigma_{g}$ along the internuclear axis $\left(\cos \theta_{\mathbf{p}} \approx 1\right)$ is constructive only for low-energy photoelectrons, whereas the interference is always constructive for all photoelectrons emitted from $1 \sigma_{g}$ along a transverse direction, for which the total photoelectron emission from $1 \sigma_{g}$ in $\mathrm{H}_{2}$ is to be predominant. However, for the case of strongly aligned diatomics, the transverse directions correspond to the most weak EM coupling to laser field that eventually leads to a poor total ionization of $\mathrm{H}_{2}$ compared to companion Ar. Finally, for the $\mathrm{N}_{2}$ molecule, the proposed SFA-LCAO model predicts no suppression (or even some enhancement compared to companion Ar) in ionization mostly due to a considerably prolated spatial configuration of the outermost $3 \sigma_{g}$ MO whose two $2 p_{z}$ AOs are oriented (and, thus, most easily ionized) along the internuclear axis, for which, in addition, the EM coupling to laser field is the strongest for the case of molecular alignment under consideration. Moreover, for ionization of $3 \sigma_{g} \mathrm{MO}$, the intramolecular interference along the internuclear axis is constructive only for low-energy photoelectrons, although, predominantly contributing to the respective ionization rate. Besides that, at a very high laser intensity, some minor en- hancement in ionization of $\mathrm{N}_{2}$ is also partly caused by an additional comparable contribution from ionization of other (inner) valence shells.

\section{ACKNOWLEDGMENTS}

The authors are indebted and very grateful to Professor Howard R. Reiss, Dr. Wilhelm Becker, and Professor Valentin N. Ostrovsky for their valuable remarks and helpful and stimulating discussions. This work was supported by the Chemical Sciences, Geosciences, and Biosciences Division of the Office of Basic Energy Sciences, Office of Science, U.S. Department of Energy. Vladimir I. Usachenko also acknowledges gratefully the support from DAAD (Deutscher Akademischer Austauschdienst) and would like to express his gratitude to Dr. Wolfgang Sandner and Dr. Wilhelm Becker for the hospitality and additional support kindly extended to him from B-Division of Max-Born-Institute of Nonlinear Optics and Short-Pulse Laser Spectroscopy (Berlin, Germany). The research described in this presentation was made also possible in part by financial support from the U.S. Civilian Research and Development Foundation (CRDF) for the Independent States of the Former Soviet Union.
[1] T. Brabec and F. Krausz, Rev. Mod. Phys. 72, 545 (2000).

[2] M. Protopapas, C. H. Keitel, and P. L. Knight, Rep. Prog. Phys. 60, 389 (1997); P. Salieres et al., Adv. At., Mol., Opt. Phys. 41, 83 (1999).

[3] Proc. of ICOMP-99 Conference, edited by L. F. Di Mauro, R. R. Freeman, and K. C. Kulander, AIP Conf. Proc. No. 525 (AIP, New York, 2000).

[4] Proc. of PISA-2000 Conference, edited by N. Bloembergen, N. Rahman, and A. Rizzo (Societe Italiana di Fisica, Bologna, 2001), Vol. 71.

[5] D. B. Milosevic and F. Ehlotzky, Adv. At., Mol., Opt. Phys. 49, 373 (2003).

[6] J. H. Eberly, J. Javanainen, and K. Rzazewski, Phys. Rep. 204, 331 (1991); L. F. DiMauro and P. Agostini, Adv. At., Mol., Opt. Phys. 35, 79 (1995).

[7] W. Becker, F. Grasbon, R. Kopold, D. B. Milosevic, G. G. Paulus, and H. Walther, Adv. At., Mol., Opt. Phys. 48, 36 (2002).

[8] H. R. Reiss, Prog. Quantum Electron. 16, 1 (1992); Phys. Rev. A 42, 1476 (1990).

[9] C. J. Joachain, M. Dörr, and N. Kylstra, Adv. At., Mol., Opt. Phys. 42, 225 (2000).

[10] A. Assion et al., Science 282, 919 (1998); R. J. Levis et al., Science 292, 709 (2001).

[11] M. J. DeWitt et al., Chem. Phys. 218, 211 (1997); S. M. Hankin, D. M. Villeneuve, P. B. Corkum, and D. M. Rayner, Phys. Rev. Lett. 84, 5082 (2000).

[12] S. L. Chin, Y. Liang, J. E. Decker, F. A. Ilkov, and M. V. Ammosov, J. Phys. B 25, L249 (1992); T. D. G. Walsh, J. E. Decker, S. L. Chin, J. Phys. B 26, L85 (1993); T. D. G. Walsh, F. A. Ilkov, J. E. Decker, S. L. Chin, J. Phys. B 27, 3767
(1994)

[13] A. Talebpour, C.-Y. Chien, S. L. Chin, J. Phys. B 29, L677 (1996); A. Talebpour, S. Larochelle, and S. L. Chin, J. Phys. B 31, L49 (1998).

[14] C. Guo, M. Li, J. P. Nibarger, and G. N. Gibson, Phys. Rev. A 58, R4271 (1998).

[15] M. J. DeWitt, E. Wells, and R. R. Jones, Phys. Rev. Lett. 87, 153001 (2001)

[16] E. Wells, M. J. DeWitt, and R. R. Jones, Phys. Rev. A 66, 013409 (2002)

[17] A. M. Perelomov, V. S. Popov, M. V. Terent'ev, Sov. Phys. JETP 23, 924 (1966).

[18] M. V. Ammosov, N. B. Delone, and V. P. Krainov, Sov. Phys. JETP 64, 1191 (1986).

[19] L. V. Keldysh, Zh. Eksp. Teor. Fiz. 47, 1945 (1964) [Sov. Phys. JETP 20, 1307 (1965); F. Faisal, J. Phys. B 6, L312 (1973). H. R. Reiss, Phys. Rev. A 22, 1786 (1980).

[20] X. Chu and Shih-I Chu, Phys. Rev. A 63, 013414 (2000).

[21] A. Saenz, J. Phys. B 33, 4365 (2000).

[22] J. Muth-Böhm, A. Becker, and F. H. M. Faisal, Phys. Rev. Lett. 85, 2280 (2000); A. Jaron-Becker, A. Becker, and F. H. M. Faisal, Phys. Rev. A 69, 023410 (2004).

[23] K. Mishima, M. Hayashi, J. Yi, S. H. Lin, H. L. Selzle, and E. W. Schlag, Phys. Rev. A 66, 033401 (2002).

[24] X. M. Tong, Z. X. Zhao, and C. D. Lin, Phys. Rev. A 66, 033402 (2002)

[25] H. D. Cohen and U. Fano, Phys. Rev. 150, 30 (1966).

[26] M. Lein et al., Phys. Rev. A 66, 023805 (2002); R. Kopold, W. Becker, and M. Kleber, ibid. 58, 4022 (1998).

[27] T. K. Kjeldsen and L. B. Madsen, J. Phys. B 37, 2033 (2004).

[28] V. S. Popov, Usp. Fiz. Nauk 169, 819 (1999) [Phys. Uspekhi 
42, 733 (1999)].

[29] N. B. Delone and V. P. Krainov, "Atoms in Strong Light Fields," Springer Ser. Chem. Phys. Vol. 28 (Springer-Verlag, Berlin, 1985).

[30] V. I. Usachenko, V. A. Pazdzersky, and J. K. McIver, Phys. Rev. A 69, 013406 (2004).

[31] T. Seideman, Annu. Rev. Phys. Chem. 53, 41 (2002).

[32] H. Stapelfeldt and T. Seideman, Rev. Mod. Phys. 75, 543 (2003).

[33] S. M. Hankin, D. M. Villeneuve, P. B. Corkum, and D. M. Rayner, Phys. Rev. A 64, 013405 (2001); J. J. Larsen, K. Hald, N. Bjerre, H. Stapelfeldt, and T. Seideman, Phys. Rev. Lett. 85, 2470 (2000); B. Freidrich and D. Herschbach, ibid. 74, 4623 (1995).

[34] M. J. DeWitt, B. S. Prall, and R. J. Lewis, J. Chem. Phys. 113, 1553 (2000).

[35] D. Normand, L. A. Lompre, and C. Cornaggia, J. Phys. B 25, L497 (1992); P. Dietrich, D. T. Strickland, M. Laberge, and P.
B. Corkum, Phys. Rev. A 47, 2305 (1993); B. Zon and B. G. Katsnelson, Sov. Phys. JETP 42, 595 (1970).

[36] S. Banerjee, G. R. Kumar, and D. Mathur, Phys. Rev. A 60, R3369 (1999).

[37] J. H. Posthumus, J. Plumridge, M. K. Thomas, K. Codling, L. J. Frasinski, A. J. Langley, and P. F. Taday, J. Phys. B 31, L553 (1998).

[38] C. Elert and P. B. Corkum, Phys. Rev. A 59, R3170 (1999).

[39] A. Giusti-Suzor, F. H. Hies L. F. DiMauro, E. Charron, and B. Yang, J. Phys. B 28, 309 (1995).

[40] B. Rotenberg, R. Taieb, V. Veniard, and A. Maquet, J. Phys. B 35, L397 (2002).

[41] B. Chelkowsky, C. Foisy, and A. D. Bandrauk, Phys. Rev. A 57, 1176 (1998).

[42] F. Grasbon, G. G. Paulus, S. L. Chin, H. Walther, J. MuthBöhm, A. Becker, and F. H. M. Faisal,Phys. Rev. A 63, 041402(R) (2001). 